\title{
Kapitel 7: Theorieunabhängige perzeptuelle Beobachtungen
}

\section{Einleitung}

Perzeptuelle Beobachtungen sind weitgehend theorieunabhängig. Diese These soll in diesem Kapitel verteidigt werden. Hierzu ist zu zeigen, dass die anführbaren Begründungen der Theoriebeladenheit bei perzeptuellen Beobachtungen weitgehend ins Leere laufen und es stattdessen gute Gründe für deren Theorieunabhängigkeit gibt. Dies soll im Folgenden nacheinander für verschiedene Arten perzeptueller Beobachtungen und die möglichen Begründungen ihrer Theorieabhängigkeit geschehen.

Bei den perzeptuellen Beobachtungen unterscheide ich zwischen direkten Wahrnehmungen (Abschn. 2 bis 4), Wahrnehmungen mit Hilfe von Instrumenten (Abschn. 5) und Wahrnehmungen auf Bildern, die mit bildgebenden Verfahren produziert werden (Abschn. 6). Es ist jeweils zu argumentieren, dass die Begründungen der Theoriebeladenheit, die sich in den Kapiteln 3 bis 5 als vorläufig vertretbar herausgestellt haben, letztlich nicht greifen. So wird es hauptsächlich darum gehen, ob erstens die Verarbeitung der Sinnesreize, die zu Sinneserfahrungen, perzeptuellen Klassifikationen und Wahrnehmungsurteilen führt, von Theorien beeinflusst wird (siehe Kap. 3). Zweitens stellt sich die Frage, ob die Bedeutung von Beobachtungsausdrücken theoretisch konstituiert wird (siehe Kap. 4). Und drittens ist zu untersuchen, ob durch die wissenschaftliche Einschätzung der Verlässlichkeit von Beobachtungsprozessen deren Ergebnisse theorieabhängig werden (siehe Kap. 5).

Die Kapitel 3 bis 5 haben gezeigt, dass starke Fassungen verschiedener Theoriebeladenheitsthesen ausreichen, um einen Pessimismus zu begründen und so die Objektivität von Beobachtungen in Zweifel zu ziehen. In Kapitel 6 wurde nachgewiesen, dass perzeptuelle Beobachtungen in der empirischen Stützung unverzichtbar sind. Die Theorieunabhängigkeit perzeptueller Beobachtungen ist daher wichtig für die Objektivität von Beobachtung insgesamt. Es soll aber erst im nächsten Kapitel gezeigt werden, dass diese Objektivität trotz der verbleibenden Theoriebeladenheit insbesondere von anderen Beobachtungsarten als perzeptuellen Beobachtungen besteht.

\section{Perzeptuelle Reizverarbeitung}

Direkte Wahrnehmungen führen ohne den Einsatz von Instrumenten von den Objekten der Wahrnehmung über Sinnesreize und deren Verarbeitung zu Sinneserfahrungen und Wahrnehmungsurteilen, die für eine weitere kognitive Verwendung etwa in expliziten Schlüssen zur Verfügung stehen. Dabei sind Objekte in der Sinneserfahrung nicht nur als mit sinnlichen Eigenschaften - 
etwa als von bestimmter Farbe und Gestalt -, sondern auch als mit reichhaltigen alltäglichen oder wissenschaftlichen Eigenschaften repräsentiert. Mir kommen in der Regel in der Sinneserfahrung Objekte unmittelbar als Amsel, als aus Holz oder als mein Freund Paul vor. Der Wahrnehmungsprozess führt zu solchen perzeptuellen Klassifikationen, die Teil der bewussten Erfahrung sind, und zu entsprechenden Wahrnehmungsurteilen. (Siehe Kap. 3, Abschn. 2 u. 3.) Es stellen sich die Fragen, ob die Reizverarbeitung, die perzeptuelle Klassifikation, deren begriffliche Inhalte oder die Einschätzung der Verlässlichkeit der Wahrnehmungen theorieabhängig sind.

\section{a) Helmboltz'sche Theorien versus modulare Theorien der Wabrnebmung}

In Kapitel 3 wurde deutlich, dass sich Vertreter der Theorieabhängigkeit von Wahrnehmungen zentral auf eine Helmholtz'sche Wahrnehmungstheorie berufen. Einer typischen Helmholtz'schen Position zufolge ist der visuelle Eindruck bzw. die visuelle Klassifikation Ergebnis einer Verarbeitung der Reize in Schlüssen, die meist unbewusst bleiben. (Wie in Kapitel 3 werde ich hier visuelle Wahrnehmung stellvertretend für Wahrnehmung insgesamt behandeln.) In diese Verarbeitung soll - in Abhängigkeit von früherer Erfahrung und Lernen - sehr verschiedenes weiteres Wissen einfließen können. Man nimmt zudem an, dass das Vokabular, in dem dieses Wissen formuliert ist, keiner natürlichen Beschränkung unterliegt, so dass auch theoretisches und theorieabhängiges Wissen in die Verarbeitung eingehen können. Schließlich soll die Verarbeitung intelligent sein in der Weise, wie es Problemlösen ist. Es soll möglich sein, verschiedene Strategien der Verarbeitung zu verfolgen und alternative Zwischenergebnisse abzuwägen. ${ }^{1}$ Insgesamt sieht die Helmholtz'sche Theorie die Verarbeitung des visuellen Reizes daher als sehr ähnlich zu zentralen Denkvorgängen, es gibt demnach keinen kategorialen Unterschied zwischen der Verarbeitung in der Wahrnehmung und in allgemeiner Kognition. Wie in Kapitel 3 gesehen, legt eine solche Theorie etwa in der Kuhn'schen Ausarbeitung nahe, dass verschiedene Beobachter, deren Wissen und perzeptuelle Verarbeitung durch unterschiedliche theoretische Hintergründe geprägt sind, in gleichen Situationen oft zu sehr verschiedenen Wahrnehmungen - etwa perzeptuellen Klassifikationen - kommen.

$\mathrm{Zu}$ dieser Helmholtz'schen Grundposition gibt es aber seit den 1980er Jahren eine gewichtige theoretische Alternative, die insbesondere von David Marr, Jerry Fodor und Zenon Pylyshyn vertreten wird:2 die ,modulare Theorie des Sehens. Dabei setzen sie zwar auch voraus, dass der Wahrneh-

1 Vgl. Kap. 3, Abschn. 4b(iii).

2 Siehe Marr (1982), Fodor (1983) und (1989), Pylyshyn (1999); vgl. Kitcher (1985). 
mungsprozess die Verarbeitung von Symbolen oder Repräsentationen einschließt und insofern aus (unbewussten) Schlüssen besteht. Aber im Prinzip ist eine modulare Theorie nicht darauf festgelegt und könnte wohl auch im Rahmen einer konnektionistischen Auffassung mentaler Verarbeitung formuliert werden. Denn zentral für die modulare Theorie ist erstens, dass die Verarbeitung nicht als intelligent in der Art allgemeinen Problemlösens aufgefasst wird. Vielmehr soll die Verarbeitung endogen feststehenden Abläufen folgen. Anders als bei intelligentem Problemlösen ist die Verarbeitungsprozedur nicht variabel und muss nicht erst noch festgelegt werden. ${ }^{3}$ Zweitens läuft die Verarbeitung abgeschlossen von anderen, insbesondere zentralen kognitiven Prozessen und Informationen ab. Dem visuellen System stehen demnach keine Informationen oder Informationen nur in sehr beschränktem Umfang aus diesen anderen Bereichen zur Verfügung, und die Zwischenschritte der visuellen Verarbeitung sind außerhalb des visuellen Systems nicht verfügbar. Das Wissen, das in die Verarbeitung einfließt, soll drittens in einem beschränkten Vokabular formuliert sein. So sollen vorwiegend Annahmen über geometrische Eigenschaften von Gegenständen verwendet werden. Die Verarbeitung im visuellen System verläuft somit modular, das visuelle System stellt ein Modul dar, das von den anderen Bereichen abgekapselt ist. Der Unterschied zwischen Kognition im Allgemeinen und Wahrnehmung wird so rehabilitiert.

Diese Position hat zur Konsequenz, dass die Ergebnisse der visuellen Verarbeitung nicht stark theorieabhängig sind. Die Outputs eines modularen visuellen Systems können nicht vor dem Hintergrund verschiedener Theorien grundlegend verschieden sein. Denn erstens besteht der Input des visuellen Systems in den Sinnesreizen, die als solche theorieneutral sind. Zweitens hat das System selbst nur sehr beschränkte Ressourcen, die nicht durch zentrale theoretische Annahmen oder theorieabhängige Ausbildung prinzipiell variabel sind. Und drittens ist die Verarbeitungsweise starr festgelegt und lässt so ebenfalls keinen Freiraum zu.

In den beiden folgenden Unterabschnitten will ich diskutieren, auf welche Weise diese Theorieunabhängigkeit der Ergebnisse visueller Verarbeitung zu verstehen ist, wie sich diese Ergebnisse zu visuellen Erfahrungen und insbesondere perzeptuellen Klassifikationen verhalten und wie die empirische Datenlage aussieht. Da sich trotz der Übereinstimmung in der Grundposition doch einige relevante Abweichungen zwischen den genannten Vertretern der modularen Theorie finden, werde ich mich hierbei auf einen Autor konzentrieren. Ich werde Pylyshyn (1999) heranziehen. Seine Position ist exempla-

3 Siehe Fodor (1989), 212-214. 
risch insoweit, als er wesentliche Einsichten der anderen beiden Autoren aufnimmt. Zugleich nimmt er auch eine Einschätzung der umfangreichen empirischen Evidenz vor.

\section{b) Die kognitive Abgeschlossenheit des visuellen Systems}

Der Wahrnehmungsprozess führt letztlich zu perzeptuellen Erfahrungen bzw. zu perzeptuellen Klassifikationen oder Wahrnehmungsurteilen. Dabei werden die Objekte auch gemäß reichhaltigen alltäglichen oder wissenschaftlichen Kategorien für Arten, Eigenschaften oder Individuen klassifiziert. Pylyshyn argumentiert dafür, dass ein wichtiger Teil dieses Wahrnehmungsprozesses modular - oder wie er es formuliert -, kognitiv abgeschlossen bzw. undurchdringlich (cognitively impenetrable) oder kognitiv abgekapselt (cognitively encapsulated) abläuft. Diesen Abschnitt des Prozesses bezeichnet Pylyshyn als frühes Sehen (early vision), das entsprechende Modul als early vision system oder einfach als das visuelle System. Auf anderen Stufen des Wahrnehmungsprozesses als dem so verstandenen visuellen System hält Pylyshyn dagegen kognitive Einflüsse für möglich.

Der Input des visuellen Systems soll aus den Reizen vor allem der Retina, aber wohl auch des Gleichgewichtsorgans und aus proprioperzeptiven Reizen der Augen bestehen. Welche Reize hereinkommen, wird dadurch beeinflusst, worauf sich die Aufmerksamkeit richtet. Die Ausrichtung des Fokus der Aufmerksamkeit kann Pylyshyn zufolge sowohl durch explizite Erwartungen und Annahmen als auch durch Lernprozesse und Spezialisierungen beeinflusst werden. ${ }^{4}$

Die Verarbeitung der Reize im visuellen System soll dann ausschließlich den modular festgelegten Prinzipien folgen. Demnach sind die Schlussweisen nicht durch Zustände von außerhalb des Systems beeinflussbar. Es können nur Informationen einfließen, die sich aus den Reizen oder einem lokalen, dem Modul zugehörigen Gedächtnis ergeben. Ein solches Modul kann einige weitere Merkmale aufweisen. So kann es erstens innerhalb des Moduls zu topdown-Verarbeitung kommen. Spätere Stufen der Verarbeitung, die etwa übergreifende Merkmale des visuellen Feldes betreffen, können so auf frühere Stadien zurückwirken, etwa auf lokale Inhalte der Repräsentation.

Zweitens wird angenommen, dass sog. ,natural constraints“ eine zentrale Rolle spielen. ${ }^{5}$ Darunter versteht man allgemeine geometrische oder optische Annahmen über die Beschaffenheit von Wahrnehmungsobjekten oder Wahrnehmungssituationen. Diese Annahmen sollen aber nicht explizit

\footnotetext{
4 Siehe Pylyshyn (1999), 360.

5 Vgl. Marr (1982), 103ff.
} 
repräsentiert sein. Stattdessen soll der Ablauf der modularen Verarbeitung so festgelegt sein, als würde die Verarbeitung diesen Annahmen folgen. Durch solche natural constraints kann etwa der kinetische Tiefeneffekt erklärt werden. Dieser Effekt ergibt sich beispielsweise, wenn man eine Anzahl sich bewegender Lichtpunkte auf einem Computerschirm betrachtet. Wenn die Punkte sich solchermaßen synchron bewegen, dass sie auf der Oberfläche eines dreidimensionalen starren Körpers liegen könnten, hat man auch einen entsprechenden visuellen Eindruck: Man sieht einen solchen dreidimensionalen starren Körper. Dieser Effekt lässt sich erklären, wenn man davon ausgeht, dass die modulare Verarbeitung der impliziten Annahme folgt, dass synchron bewegte Punkte zur Oberfläche starrer Körper gehören. ${ }^{6}$

Es wird drittens für plausibel gehalten, dass das visuelle System intern in weitere Subsysteme unterteilt ist, die einzelne Merkmale wie Farbe, Form, Bewegung, Helligkeit oder dreidimensionale Ausrichtung bei nur beschränktem Austausch untereinander berechnen.

Der Output des visuellen Systems soll Pylyshyn zufolge noch nicht in der Sinneserfahrung und den eingeschlossenen perzeptuellen Klassifikationen bestehen. Vielmehr wird angenommen, dass nur eine Vorstufe für solche Klassifikationen herauskommt. Im Output sollen insbesondere sichtbare Formen und Oberflächen im Raum repräsentiert werden, die in einem geometrischen Vokabular beschreibbar sind.7 Obwohl es empirisch nicht völlig klar ist, welche Inhalte die Ergebnisse der visuellen Verarbeitung genau haben, spricht einiges dafür, dass die Aufschlüsselung von Form und Ausrichtung der Oberflächen zueinander und in Bezug auf den Beobachter auf der Basis von Repräsentationen solcher Eigenschaften wie Farbe, Helligkeit und Bewegung geschieht. Möglicherweise enthält der Output auch einige besondere Merkmale wie die wahrgenommene Gefährlichkeit oder einfache kausale Relationen. ${ }^{8}$ Diese Eigenschaften bezeichne ich im Folgenden als ,modular repräsentierbar'. Es ist klar, dass diese modular repräsentierbaren Eigenschaften noch nicht den unmittelbaren Inhalt bewusster Erfahrungen und deren Klassifikation gemäß gewöhnlicher perzeptueller Kategorien ausschöpfen. Pylyshyn zufolge beruht diese perzeptuelle Klassifikation allerdings auf der modularen Repräsentation. Da er aber davon ausgeht, dass diese Klassifikation auf Wissen zurückgreifen kann, das einem Modul nicht

6 Siehe Pylyshyn (1999), 354.

7 Siehe Pylyshyn (1999), 361. Dies entspricht Marrs 21/2-D-Skizze. Siehe Marr (1982), Kap. 4.

8 Siehe Pylyshyn (1999), 360/361. 
zur Verfügung steht, zählt er diesen Schritt nicht mehr als Teil der Verarbeitung des visuellen Systems:?

$[T]$ he visual system does not identify the stimulus in the sense of cross-referencing it to the perceiver's knowledge base ... That is because the category identity is inextricably linked to past encounters and to what one knows about members of the category (e.g., what properties - visual and nonvisual - they have). After all, identifying some visual stimulus as your sister does depend on knowing such things as that you have a sister, what she looks like, whether she recently dyed her hair, and so on. But, according to the present view, computing what the stimulus before you looks like - in the sense of computing some representation of its shape, sufficient to pick out the class of similar appearances - and hence to serve as an index into long-term memory - does not itself depend on knowledge. According to this view, the visual system is seen as generating a set of one or more shapedescriptions that might be sufficient (perhaps in concert with other contextual information) to identify objects stored in memory. (Pylyshyn 1999, 361)

Gemäß der modularen Theorie des Sehens, wie sie von Pylyshyn vertreten wird, ist daher die visuelle Verarbeitung der Reize bis hin zu einer Repräsentation der sichtbaren Oberflächen im Raum modular. Die perzeptuelle Klassifikation findet dann auf der Grundlage dieser modularen Repräsentation statt. Bevor ich in Abschnitt 3 zu diesem Schritt komme, ist zu fragen, ob die empirischen Befunde eher die Helmholtz'sche oder die modulare Theorie des Sehens bestätigen.

\section{c) Empirische Belege für die kognitive Abgeschlossenheit}

Es gibt eine sehr große Zahl von Befunden und Studien, die für die konkurrierenden Theorien des Sehens relevant sind. Pylyshyn gibt einen Überblick

9 Im Gegensatz hierzu geht Fodor davon aus, dass der Output des modularen visuellen Systems auch Repräsentationen alltäglicher Kategorien umfasst, insbesondere Rosch'sche basic-level categories. Zudem soll der modulare Output Fodor zufolge bewusst als Erfahrung zugänglich sein. (Siehe Fodor 1983, 87 u. 93-97; 1988, 197.) Fodor muss dann davon ausgehen, dass Modul-externes Wissen keinen Einfluss auf diese perzeptuellen Klassifikationen ausüben kann. Ich werde im Folgenden Pylyshyn folgen und einen solchen Einfluss nicht ausschließen.

Richard Schantz, der auch eine modulare Theorie der Wahrnehmung vertritt, hält wie Fodor die Ergebnisse der modularen Verarbeitung für bewusst erfahrbar, glaubt aber im Gegensatz zu Fodor, dass deren Inhalte nicht-begrifflich sind. (Siehe Schantz 2000, 67-69.) Eine vergleichbare Position vertritt auch Bermudez (1999). Wie oben dargelegt, werde ich die Möglichkeit nicht-begrifflicher Wahrnehmungsinhalte für die Argumentation gegen die Theoriebeladenheit nicht verfolgen. (Siehe Kap. 3, Abschn. 2b.) Siehe auch Pylyshyn (1999), 362 und (1999a), 407 für Kritik an der Auffassung, dass bewusste Sinneserfahrungen direkte Hinweise auf die Inhalte der modularen Outputs liefern oder gar mit den modularen Outputs gleichzusetzen sind. 
über diese Befunde, der zeigt, dass die empirische Evidenz insgesamt für die modulare und gegen die Helmholtz'sche Theorie spricht. ${ }^{10}$ Aus der Vielzahl der Belege und Fälle möchte ich nur einige anführen.

Es gibt interessante Fälle visueller Agnosie, die dafür sprechen, dass die einzelnen Fähigkeiten und Stufen perzeptueller Verarbeitung voneinander unabhängig sind. So hat ein Patient nach einem Schlaganfall zwar die Fähigkeit behalten, einzelne Merkmale und Eigenschaften von Gegenständen zu sehen. Allerdings hat er vollkommen die Fähigkeit verloren, die Dinge als Ganze zu sehen und visuell zu erkennen, worum es sich handelt. Dennoch wusste er weiterhin, welche Merkmale Gegenstände charakterisieren und konnte die Gegenstände beispielsweise aus dem Gedächtnis zeichnen. Dieser Fall spricht dafür, dass es für die visuelle Verarbeitung nicht ausreicht, Wissen über die Eigenschaften von Dingen im Rahmen einer allgemeinen Problemlöseaufgabe mit Reizen und wahrgenommenen Teilaspekten zusammenzubringen. Vielmehr scheint eine spezifische Fähigkeit visueller Integration notwendig, die der Patient verloren hat. ${ }^{11}$

Auch viele der einfachen Effekte und Regularitäten, die sich in der Wahrnehmung finden, stützen eine modulare Theorie besser als eine Helmholtz'sche. Wie schon in Kapitel 3 erwähnt, sind viele Wahrnehmungstäuschungen wie die Müller-Lyer-Täuschung oder illusorische Konturen, wie sie sich beim Kanisza-Dreieck zeigen (Abb. 3.5), durch Wissen um ihren täuschenden Charakter unbeeinflussbar. ${ }^{12}$ Dies ist gerade zu erwarten, wenn das visuelle System autonom und von allgemeinem Wissen abgekapselt arbeitet. Vor einem Helmholtz'schen Hintergrund, wo solche Einflüsse im Prinzip erwartbar sind, ist diese Unbeeinflussbarkeit dagegen rästelhaft.

Ähnliches gilt für Regularitäten wie das gestaltpsychologische Gesetz der Nähe, wonach näher beeinander liegende Elemente eher als zur selben Gestalt gehörend wahrgenommen werden, oder wie die Weise, in der verdeckt erscheinende Figuren vervollständigt werden. Hier folgt die Wahrnehmung eigenen Regeln, die oft nicht solchen Regeln entsprechen, die zu einer rational optimalen Lösung führen würden. Dies zeigt sich in Abb. 7.1. Die mittlere Figur wird zu einem unregelmäßigen Sechseck vervollständigt, obwohl der Kontext der anderen beiden Figuren nahe legt, dass es sich auch hier um ein regelmäßiges Achteck handelt. ${ }^{13}$ Dies stützt gerade die Annahme,

10 Siehe insbesondere auch die im Anschluss an Pylyshyn (1999) abgedruckten Kommentare für teilweise übereinstimmende, teilweise abweichende Einschätzungen der empirischen Evidenzlage.

11 Siehe Humphreys / Riddoch (1987); vgl. Pylyshyn (1999), 348.

12 Siehe Rock (1984), 228 u. (1985), 4.

13 Siehe Kansiza (1985), 30ff.; vgl. Pylyshyn (1999), 344/345. 
dass der Reiz starr, informativ abgeschirmt und nicht intelligent verarbeitet wird.

\section{Abb. 7.1}
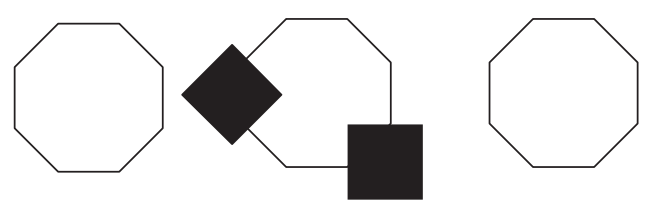

Was solche Fälle betrifft, kommt einer der Hauptvertreter der Helmholtz'schen Position, Irvin Rock, in seiner Ausarbeitung der Position der modularen Theorie weit entgegen. ${ }^{14}$ So geht Rock wie Pylyshyn davon aus, dass die visuelle Wahrnehmung in zwei Hauptschritten erfolgt. Zunächst werde eine Repräsentation erzeugt, die visuelle Eigenschaften wie die Form und die räumliche Lage der Objekte enthalte. Dann erst würden Gegenstände klassifiziert. Erst für den zweiten Schritt sei konkretes Wissen über die wahrgenommenen Objekte notwendig, das aus früherer Erfahrung stamme. Der erste Schritt sei dagegen sowohl von explizitem, bewusstem Wissen als auch von implizitem Wissen über die spezifischen Eigenschaften und Arten unabhängig.

Anders als die modulare Theorie betont Rock aber die Intelligenz auch der ersten Stufe der Wahrnehmung in solchen Fällen, in denen komplexere Regularitäten betroffen sind. Hierzu zählen insbesondere die Wahrnehmungskonstanzen. Beispielsweise können zwei unterschiedlich helle Hälften einer Seite als gleich hell (d.h. als von gleichem Grauton) erscheinen. So könnte die eine Hälfte weiß und die sich anschließende Hälfte grau sein und dennoch die Seite ganz als weiß erscheinen. Um diesen Effekt zu erzielen, muss die Seite so gefaltet sein, dass der Unterschied der Helligkeit auch auf einen unterschiedlichen Lichteinfall zurückgehen könnte. Die Situation muss es als möglich erscheinen lassen, dass die vergleichsweise geringere Lichtreflektion der grauen Hälfte dadurch zustande kommt, dass sie beispielsweise im Schatten liegt. Dieser Effekt lässt sich erklären, wenn man annimmt, dass das visuelle System die Möglichkeiten unterschiedlicher Beleuchtung der Hälften ,in Betracht zieht ${ }^{\varsigma}$ und daraus folgert, dass die Seiten tatsächlich dieselbe Helligkeit (denselben Grauton) haben. ${ }^{15}$

Bei der Wahrnehmung der Größe von Objekten spielt insbesondere die wahrgenommene Entfernung eine Rolle. Es ist ein einfaches geometrisches Gesetz, dass der Sehwinkel, unter dem ein Objekt erscheint, umgekehrt

14 Siehe Rock (1985), insbes. 16.

15 Vgl. Rock (1984), 49; Churchland (1988), 172; Pylyshyn (1999), 355. 
proportional zur Entfernung des Objekts ist. Diese Regel scheint sich das visuelle System zunutze zu machen, um die Größe von Objekten zu bestimmen. Dies zeigt sich beispielsweise an der scheinbaren Größe von Nachbildern. Ein bestimmtes Nachbild erscheint umso größer, je weiter die betrachtete Fläche entfernt ist. ${ }^{16}$

Allerdings sprechen auch diese Beispiele scheinbaren intelligenten Schlussfolgerns in der Wahrnehmung letztlich eher für die modulare Theorie als für die Helmholtz'sche Theorie. Denn die herangezogenen Regeln sind gerade von solcher Art, von der die natural constraints gemäß der modularen Theorie sind. ${ }^{17}$ Sie betreffen optische oder geometrische Eigenschaften, die innerhalb des visuellen Systems verfügbar sind - etwa scheinbare Entfernung oder Beleuchtung. Zudem gelten sie allgemein und nicht etwa nur für besondere Fälle. Es hängt daher nicht an intelligentem Problemlösen, ob sie eingesetzt werden. Damit können sie in einer autonomen und informational abgekapselten Reizverarbeitung implizit wirksam werden. Doch damit steht nicht nur eine Erklärung der Wahrnehmungskonstanzen zur Verfügung, die der Helmholtz'schen Theorie gleichwertig wäre. Die modulare Theorie braucht für diese Erklärung nur mentale Mechanismen anzunehmen, die niedrigstufiger als bei der Helmholtz'schen Erklärung sind. Statt von intelligentem Problemlösen mit potenziellem Zugriff auf vielfältige, in reichhaltigem Vokabular formulierte Regeln auszugehen, braucht bloß eine starre Verarbeitung nach implizit verkörperten Regularitäten in beschränktem Vokabular angenommen $\mathrm{zu}$ werden. Eine solche sparsamere Erklärung ist aber zu bevorzugen. Die modulare Theorie ist deshalb auch hier der Helmholtz'schen überlegen. ${ }^{18}$

Schließlich verfügt die modulare Theorie auch über die Ressourcen, um ein Problem, das oft im Zentrum des Interesses Helmholtz'scher Theorien steht, zu erklären. Wie schafft das visuelle System es, zu einer eindeutigen und oft zutreffenden Repräsentation der wahrgenommenen Objekte und Eigenschaften zu kommen, obwohl der Reiz nur unzureichend informativ ist? ${ }^{19}$ Eine besondere Schwierigkeit stellt die sog. inverse Abbildung dar, d.h. die Erzeugung einer dreidimensionalen visuellen Repräsentation auf der Basis

16 Vgl. Rock (1984), 29ff. Das Nachbild entsteht dadurch, dass die Rezeptoren in einem bestimmten Bereich der Netzhaut durch eine vorausgehende Reizung übermüdet sind und nun beim Betrachten einer homogenen Fläche ein anderes Signal senden als die benachbarten Rezeptoren. Die unterschiedlich groß erscheinenden Nachbilder beruhen daher auf der Reizung eines konstanten Ausschnitts der Netzhaut, entsprechend einem konstanten Sehwinkel.

17 Siehe Pylyshyn (1999), 355/356.

18 Siehe Pylyshyn (1999), 357; vgl. Rock (1983), 338/339.

19 Vgl. Kap. 4, Abschn. 4b(iii). 
von bloß zweidimensionalen Reizen. Auch hier geht die modulare Theorie davon aus, dass in die Verarbeitung eingebaute natural constraints eine zentrale Rolle spielen. Auf solche Weise kann man durch Bezug auf die oben angeführte Annahme, dass synchron bewegte Punkte auf der Oberfläche starrer Körper liegen, nicht nur den kinetischen Tiefeneffekt erklären. Mit dieser Annahme lassen sich auch die relativen Positionen mehrerer Punkte im Raum eindeutig aus einer kurzen Sequenz zweidimensionaler Bilder dieser bewegten Punkte bestimmen. Wenn die Verarbeitung der Reize dieser Annahme folgt, kommt das visuelle System daher zu einer eindeutigen Repräsentation. Die modulare Theorie des Sehens kann so die Fähigkeit zur inversen Abbildung erklären. ${ }^{20}$

Auch hierbei ist die modulare Erklärung besser empirisch gestützt als die Helmholtz'sche. So stellt sich der kinetische Tiefeneffekt ein, obwohl man die bewegten Punkte auf einem Computerschirm betrachtet, es also dem Betrachter klar ist, dass die Punkte nicht auf einem starren Körper liegen. ${ }^{21}$ Wieder zeigt sich die Verarbeitungsweise des visuellen Systems daher als durch Wissen des Beobachters über die Situation unbeeinflussbar. Das visuelle System ist inflexibel und folgt bloß seinen eigenen Prozeduren.

Die modulare Theorie erweist sich damit insgesamt der Helmholtz'schen Theorie überlegen. Sie kommt zum einen mit dem Postulieren von niedrigstufigeren mentalen Mechanismen aus. So werden die perzeptuellen Fähigkeiten durch einfache und inflexible Reizverarbeitung erklärt. Eine Helmholtz'sche Theorie muss demgegenüber eine anspruchsvolle Verarbeitungsweise annehmen, die auf einen großen Fundus von Wissen zurückgreifen und diesen intelligent einbringen kann. Zum anderen sprechen die empirischen Befunde in ihrer Mehrzahl für die modulare Theorie, wohingegen die Helmholtz'sche Theorie in vielen Fällen eine größere Flexibilität der visuellen Reizverarbeitung und eine stärkere Beeinflussung von weiterem, im Prinzip zur Verfügung stehendem Wissen erwarten ließe.

Der modularen Theorie zufolge sind die Outputs des visuellen Systems aber weitreichend theorieunabhängig. Zwar wird dieser Output nicht ausschließlich durch den Reiz bestimmt. Vielmehr sind die natural constraints in die Verarbeitung inkorporiert. Damit fließen einige Annahmen darüber, wie

20 Siehe Pylyshyn (1999), 354. Für eine vollständige Erklärung der inversen Abbildung reicht die genannte Annahme natürlich nicht aus. Vielmehr müssen auch schon bei bewegten Objekten weitere natural constraints sowie ein Modus, gemäß dem sie interagieren, angenommen werden. Eine solche umfassende Erklärung gibt es noch nicht. Siehe hierfür Pylyshyn (1999), 355 und (1999a), 412.

21 Vgl. Pylyshyn (1999), 355. 
typische Wahrnehmungssituationen beschaffen sind, in die Wahrnehmung ein. Aber daraus ergibt sich keine Theorieabhängigkeit.

Erstens ergibt sich keine starke Theorieabhängigkeit. Denn die Verarbeitung im visuellen System kann gemäß der modularen Theorie nicht mit einem variablen theoretischen Hintergrund variieren. Vielmehr sind diese Verarbeitung und die bestimmenden natural constraints endogen festgelegt. Zweitens ergibt sich auch keine schwache Theorieabhängigkeit, etwa eine Abhängigkeit von einer angeborenen Theorie, der wir nicht entgehen könnten. Denn die einfließenden natural constraints sind nicht theoretisch in irgendeinem interessanten Sinn. Sie sind bloß in einem beschränkten, visuellen oder geometrischem Vokabular formuliert. Sie betreffen solche Faktoren wie den Sehwinkel, unter dem Objekte erscheinen, deren Entfernung, Starrheit oder Beleuchtung. Sie greifen damit den meisten Eigenschaften, nach denen perzeptuell klassifiziert werden kann, und entsprechenden, hierfür zuständigen alltäglichen oder wissenschaftlichen Theorien nicht vor. Drittens ist zu erwarten, dass eine solche Reizverarbeitung in den überwiegenden Fällen zu wahren Repräsentationen führt. Denn das visuelle System und die damit verbundene Verarbeitungsweise sind der modularen Theorie zufolge endogen festgelegt und daher evolutionär entstanden. Man kann daher davon ausgehen, dass die natural constraints an unsere gewöhnliche Umgebung angepasst sind. Da aber zutreffende Wahrnehmungen in der Regel den größten Fitnesswert besitzen, kann man davon ausgehen, dass das visuelle System auch seiner Zuverlässigkeit wegen selektiert wurde. ${ }^{22}$

Zwar geht Pylyshyn davon aus, dass auf anderen Stufen der Reizverarbeitung als der im visuellen System ein kognitiver Einfluss möglich ist. Erstens kann die Fähigkeit, den Fokus der Aufmerksamheit auszurichten, für viele Situationen erlernt werden, und zweitens ist sicherlich die perzeptuelle Klassifikation von Lernprozessen und früherer Erfahrung abhängig. Durch den ersten Einfluss - auf die Ausrichtung des Fokus - können sich in derselben Situation natürlich unterschiedliche Wahrnehmungen ergeben. So kann es kommen, dass der eine etwas wahrnimmt, das dem anderen entgeht, oder dass mehrdeutige Figuren wie der Hasen-Enten-Kopf, der Neckerwürfel oder das Bild der alten bzw. jungen Frau unterschiedlich erscheinen. ${ }^{23}$ (Siehe Abb. 3.1, 3.2 u. 3.3.) Aber auch aus einem Einfluss von Lemprozessen auf die Ausrichtung der Aufmerksamkeit kann sich letztlich keine Theorieabhängigkeit ergeben. Auch wenn man mit einer solchen, eventuell theoretisch geleiteten Ausbildung leichter zu den Stimuli kommt, die in einer bestimmten

\footnotetext{
22 Vgl. Pylyshyn (1999), 357.

23 Vgl. Pylyshyn (1999), 358.
} 
Situation für die Wahrnehmung wichtig sind, kann man diese Reize auch ohne Ausbildung erhalten. Der Input bestimmter Stimuli hängt letztlich nicht von einem theoretischen Hintergrund ab. ${ }^{24}$

Ob die zweite Art kognitiver Einflüsse - auf die perzeptuellen Klassifikationen - zu einer Theorieabhängigkeit der Wahrnehmungen führt, werde ich jetzt ausführlich untersuchen.

\section{Fähigkeiten perzeptueller Klassifikation}

\section{a) Theorieunabhängigkeit und Zuverlässigkeit von Klassifikationsfähigkeiten}

Perzeptuelle Erfahrungen schließen Klassifikationen gemäß alltäglichen oder wissenschaftlichen perzeptuellen Kategorien ein. Wenn man mit Pylyshyn von der Modularität des visuellen Systems ausgeht, müssen die Fähigkeiten zu perzeptueller Klassifikation im Anschluss an die Ergebnisse der visuellen Verarbeitung der Reize operieren. Dann operieren diese Fähigkeiten auf der Grundlage einer Repräsentation bestimmter Eigenschaften wie Form, räumliche Lage, Farbe, Bewegung etc. - der modular repräsentierbaren Eigenschaften. Für eine Identifikation vieler alltäglicher Eigenschaften, Arten oder Individuen muss man sicherlich spezifisches Wissen verwenden. Man kann eine Person nur erkennen, wenn man charakterisierende Merkmale kennt. Genauso kann man eine Amsel nur als Amsel sehen, wenn in die visuelle Verarbeitung Wissen über typische Amseleigenschaften einfließen. Solches Arten- oder Individuen-spezifisches Wissen bleibt oft so implizit wie die Fähigkeiten perzeptuellen Klassifizierens. Man kann aus der Klassifikation der Arten oder Individuen in der Erfahrung auf die Fähigkeiten und auf zugrunde liegendes Wissen schließen. ${ }^{25}$

Die Zuverlässigkeit von Wahrnehmungen hängt dann zentral an der Zuverlässigkeit der Klassifikationsfähigkeiten. In diesem Unterabschnitt ist zum einen zu fragen, unter welchen Bedingungen eine Klassifikationsfähigkeit als zuverlässig aufzufassen ist. Zum anderen muss geklärt werden, wann eine solche zuverlässige Klassifikationsfähigkeit als theorieunabhängig gelten kann. In den nächsten Unterabschnitten werde ich dann untersuchen, ob solche Klassifikationsfähigkeiten in unserer Welt und für uns möglich sind und von uns eingesetzt werden.

24 Vgl. Fodor (1988), 191.

25 Für die Theorieabhängigkeit und -unabhängigkeit ändert sich nichts Wesentliches, wenn die Klassifikationen explizit ablaufen, etwa auf der Grundlage bewusst wahrgenommener Eigenschaften. Wer zum ersten Mal eine Amsel sieht, kann etwa mit Hilfe expliziten Wissens aus einem Vogelbestimmungsbuch und auf der Grundlage von Wahrnehmungen der Form, Farbe und Bewegung des Vogels diesen als Amsel erkennen. 
Damit man über eine zuverlässige klassifikatorische Fähigkeit etwa für eine Vogelart verfügt, muss man die Exemplare keineswegs unter allen möglichen Umständen als solche erkennen und von anderen Arten unterscheiden können. Für erkenntnistheoretische Zwecke reicht es aus, wenn die Zuordnung in unserer gewöbnlichen Umgebung funktioniert. Es mag einen Freizeitpark geben, in dem eine Nachbildung des Markusplatzes von Venedig mit Taubenrobotern bestückt wird. Unsere alltägliche Fähigkeit, Tauben als solche zu erkennen, würde durch diese Taubenroboter irregeleitet und wäre damit im Freizeitpark unzuverlässig. Dies stellt aber nicht die epistemische Nützlichkeit der Fähigkeit für unsere gewöhnliche Umgebung in Frage. In unserer Fußgängerzone und im Vorgarten kommen Taubenroboter nicht vor. Deshalb reicht die Fähigkeit für den relevanten Kontext - hier unseren Alltag - aus epistemologischer Sicht völlig aus. ${ }^{26}$

Man kann nachschieben, dass die Klassifikationsfähigkeit auch dann brauchbar bleibt, wenn sich einige wenige Taubenroboter aus dem Freizeitpark in unsere gewöhnliche Umgebung verirren. Eine Klassifikationsfähigkeit für Tauben arbeitet für unseren Alltag auch zuverlässig genug, wenn sie die weit überwiegende Zahl von Tauben als solche erkennt und nur wenige NichtTauben als Tauben klassifiziert.

Hierbei muss man zwischen zwei Hinsichten unterscheiden, die mit einer Klassifikation von Dingen als $F$ verbunden sind. Zum ersten werden die Dinge hinsichtlich Art, Eigenschaft oder Identität als gleich zu und verschieden von anderen Dingen klassifiziert. Damit werden die Objekte eines Bereichs Äquivalenzklassen zugeordnet, und eine perzeptuelle Fähigkeit hierzu führt zu Repräsentationen der Objekte als gleich oder verschieden. Zum zweiten klassifiziert man die Objekte als von bestimmter Art, als unter einen bestimmten Begriff fallend - etwa den Begriff der Taube. Hierzu ist es aber notwendig, dass man über diesen Begriff auch verfügt. Die Theorieabhängigkeit der Klassifikation muss letztlich in beiden Hinsichten untersucht werden. Die mögliche Theorieabhängigkeit von Begriffsbesitz bereitet aber Probleme, die ich auf Abschnitt 4 verschieben möchte. Daher werde ich in diesem Abschnitt Klassifikationsfähigkeiten nur als Fähigkeiten, Objekte als gleich oder verschieden zu repräsentieren, betrachten. Perzeptuelle Klassifikationsfähigkeiten gelten demnach lokal als zuverlässig, wenn sie in der relevanten Umgebung ziemlich genau die richtigen Dinge als gleich oder verschieden repräsentieren.

Im Allgemeinen gilt, dass eine Klassifikationsfähigkeit theorieunabhängig ist, wenn man unabhängig von theoretischen Hintergründen über sie verfü-

26 Vgl. Goldman (1977) u. Brown (1998), 288-292. 
gen und sie einsetzen kann. Klassifikationsfähigkeiten sind nicht stark theorieabhängig, wenn es nicht mit den theoretischen Hintergründen variiert, über welche Fähigkeiten man verfügt oder welche man einsetzt. Sie sind nicht schwach theorieabhängig, wenn Besitz und Verwendung überhaupt keinen theoretischen Hintergrund voraussetzen.

Können lokal zuverlässige Klassifikationsfähigkeiten, die auf der Basis modular repräsentierbarer Eigenschaften operieren, theorieunabhängig sein? Wie gesagt, setzen solche Fähigkeiten Wissen über die repräsentierten Individuen, Arten oder Eigenschaften voraus, das in der Regel stillschweigend ist. Allerdings braucht dieses Wissen nicht theoretisch oder theorieabhängig zu sein.

Das eingesetzte Wissen wäre in relevanter Weise theoretisch, wenn es Teil einer Theorie wäre. Es wäre zudem theorieabhängig, wenn das Verfügen über dieses Wissen selbst von Theorien abhinge, beispielsweise wenn das Besitzen des Wissens das Glauben einer Theorie voraussetzt oder dadurch unmöglich gemacht wird. Theorien kann man aber dadurch minimal charakterisieren, dass sie eine Taxonomie von Dingen in einem Bereich implizieren oder gegenüber Phänomenen in diesem Bereich Erklärungskraft besitzen. Solche Erklärungskraft ergibt sich grob gesagt, wenn die Theorien generelle notwendige Zusammenhänge, insbesondere kausale und konstitutorische, postulieren und die Einordnung von Phänomenen in diese Zusammenhänge möglich machen. (Vgl. Kap. 4, Abschn. 3a.)

Eine lokal zuverlässige Fähigkeit zur Klassifikation als gleich oder verschieden muss aber nicht auf solchermaßen theoretisches oder theorieabhängiges Wissen zurückgreifen. Es kann die Annahme genügen, dass die weitaus meisten hier vorkommenden Exemplare einer Art (und weitgehend nur diese) eine Reihe von Merkmalen $\mathrm{E}_{1}$ bis $\mathrm{E}_{\mathrm{n}}$ besitzen - etwa dass Tauben und nur Tauben eine bestimmte Form und Bewegungsweise haben. Wenn die Eigenschaften $\mathrm{E}_{1}$ bis $\mathrm{E}_{\mathrm{n}}$ aber nicht zentral dafür sind, dass etwas zur Art gehört, trägt die Annahme nicht zu einer theoretischen Taxonomie der Objekte bei. Beispielsweise besagt das Wissen über das typische Aussehen von Tauben nichts über eine theoretische Taxonomie von Vogelarten, die sich vermutlich an der genauen Morphologie, an gemeinsamer Abstammung, genetischer Ähnlichkeit oder freier Kreuzung festmachen würde. Die Annahme trägt aber offensichtlich auch nichts zu notwendigen Generalisierungen und damit zu explanatorischen Theorien über Tauben, Vögel oder Lebewesen im Allgemeinen bei. Sie ist daher nicht theoretisch.

Auch das Verfügen über die Annahme braucht nicht theorieabhängig zu sein. Es wäre denkbar, dass man sie macht, ohne dass man über eine Theorie für Tauben oder Vögel verfügt; und es ist sogar vorstellbar, dass sie still- 
schweigend in eine Klassifikation einfließt, obwohl sie explizit und bewusst vertretenen Theorien widerspricht. Daher könnten das Wissen, das in die Klassifikationsfähigkeit einfließt, und damit auch die Klassifikationsfähigkeit selbst vollkommen theorieunabhängig sein.

\section{b) Die beobachterfreundliche $W$ elt}

Diese Möglichkeit von theorieunabhängigen und dennoch zuverlässigen Klassifikationsfähigkeiten besteht besonders dann, wenn Arten, Eigenschaften oder Individuen beobacbterfreundlich sind. Die Idee der Beobachterfreundlichkeit lässt sich auf der Grundlage der modularen Theorie des Sehens explizieren. Eine Art soll als beobachterfreundlich gelten, wenn eine Klassifikationsfähigkeit für sie möglich ist, die nur auf der Grundlage der modular repräsentierbaren Eigenschaften operiert und in der relevanten gewöhnlichen Umgebung zuverlässig ist. Dies ist gegeben, wenn es für die Art F eine Anzahl von Eigenschaften $\mathrm{E}_{1}$ bis $\mathrm{E}_{\mathrm{n}}$ gibt, die durch unser Wahrnehmungssystem modular repräsentierbar sind, und etwas in unserer gewöhnlichen Umgebung ziemlich genau dann ein $F$ ist, wenn es die Eigenschaften $E_{1}$ bis $\mathrm{E}_{\mathrm{n}}$ besitzt. Denn dann kann eine Zuordnung allein auf der Basis modular repräsentierter Eigenschaften der Arten lokal zuverlässig sein.

Diese Bedingungen für Beobachterfreundlichkeit kann man mindestens in zwei Hinsichten verfeinern. Erstens dürfte man von Instanzen von $F$ wohl nicht die strikte Instanziierung aller Eigenschaften $E_{1}$ bis $E_{n}$ verlangen, sondern nur die Instanziierung einer gewichteten Mindestanzahl von Eigenschaften, die für die Art charakteristisch sind. So wird es möglich, dass auch Arten, die durch Familienähnlichkeit gebildet werden oder die sich in Familienähnlichkeit der modular repräsentierbaren Eigenschaften ausdrücken, beobachterfreundlich sind.

Zweitens ist für epistemische Zwecke wohl nicht notwendig, dass Arten aufgrund einer intrinsischen Menge Art-typischer Eigenschaften auszumachen sind. Es reicht, wenn die Arten sich so in einer Anzahl von typischen Eigenschaften ausdrücken, dass die Exemplare von Exemplaren relevanter anderer Arten der gewöhnlichen Umgebung unterscheidbar sind. ${ }^{27}$

Beide Überlegungen lassen sich vielleicht am besten durch die Idee eines modularen Prototypen für die Arten ausdrücken. Der modulare Prototyp für Tauben ist ein Ding, welches die für Tauben prototypischen Eigenschaften, sofern sie modular repräsentierbar sind, am besten instanziiert. Demnach sind Arten unserer gewöhnlichen Umgebung beobachterfreundlich genau dann,

27 Vgl. Goldman (1977). 
wenn die Artzugehörigkeit in der weit überwiegenden Zahl der Fälle der größten Nähe zu den modularen Prototypen der relevanten Arten folgt.

(Im Folgenden werde ich aber der Einfachheit halber in der Regel auf die einfachere Bestimmung der Beobachterfreundlichkeit als Möglichkeit der Zuordnung auf der Grundlage der Eigenschaften $\mathrm{E}_{1}$ bis $\mathrm{E}_{\mathrm{n}}$ zurückgreifen. Die Fassung des Begriffs durch modulare Prototypen ist hierbei mit gemeint.)

So zeigt sich, dass viele alltägliche Arten, Eigenschaften und Individuen beobachterfreundlich sind. So gilt für die Vogelarten, die sich im Vorgarten tummeln - etwa Amseln, Sperlinge, Tauben, Rotkehlchen, Elstern, Grünfinken usw. -, dass Exemplare dieser Arten aufgrund von Form, Farbe und Bewegung meist zuverlässig als gleich und verschieden klassifiziert werden können. Unsere Amseln haben eine charakteristische Form, schwarzes oder graues Gefieder, springen in typischer Weise durchs Gras oder flattern weg. Aufgrund dieser Merkmale können wir sie in der Regel eindeutig als solche erkennen und von anderen Arten unterscheiden. Das gilt nicht nur für Vögel, auch andere Tierarten (Kühe, Schafe, Ziegen, Schäferhunde, Kaninchen ...), viele Pflanzen (Linde, Buche, Eiche, Fichte, Kiefer ...), Substanzen und Materialien (Metalle, Plastik, Papier, Holz, Beton, Granit, Marmor, Asphalt ...), Flüssigkeiten (Wasser, Milch, Öl ...), Früchte, geographische, meteorologische und geologische Formationen, Himmelskörper (Sonne, Mond), usw. und natürlich Personen. Der Bereich der Welt, der für direkte Wahrnehmungen beobachterfreundlich ist, umfasst einen großen Teil der alltagsrelevanten Welt eingeschlossen viele menschliche Artefakte wie Autos oder Straßenschilder und einen guten Teil der natürlichen Welt, insbesondere der Bereiche der Biologie, Geologie und Chemie, aber auch der Astronomie und Physik.

Damit ergibt sich jetzt ein grobes Bild davon, wie Fähigkeiten perzeptueller Klassifikation für viele Eigenschaften, Arten und Individuen theorieunabhängig möglich sein könnten. Die Reizverarbeitung im visuellen System führt zu einer Repräsentation modularer Eigenschaften der Objekte. Darauf greifen die perzeptuellen Klassifikationsfähigkeiten zu. In die Klassifikationen fließen Annahmen der Art „Dinge mit den modularen Eigenschaften $E_{1}$ bis $E_{n}$ gehören zur selben Kategorie $F^{\text {، }}$ ein, die selbst weder theoretisch noch theorieabhängig sind. (Siehe Abb. 7.2.) Auf solche Weise sind für beobachterfreundliche Eigenschaften, Arten und Individuen lokal zuverlässige Klassifikationen erreichbar.

Charakteristisch für dieses Bild ist die Unterscheidung zwischen den Eigenschaften, auf deren Basis die Klassifikation stattfindet, und der Eigenschaft (Art, etc.), als die besitzend das Objekt repräsentiert wird. Als Klassifikationsbasis sollen modulare Repräsentationen dienen. Hierfür sprechen die Umstände, unter denen wir uns mit perzeptuellen Klassifikationen täuschen. 
Papierblumen sehen für uns oft wie echte Blumen aus, sie sind in unserer Wahrnehmung als echte Blumen repräsentiert und klassifiziert. Eine Wachsfigur von Charly Chaplin kann uns wie Charly Chaplin erscheinen, eine PressSpanplatte, die mit einem imitierten Holzfurnier aus Plastik überzogen ist, sieht wie ein massives Brett aus. Jedesmal teilen sich echte Instanzen und Imitate modular repräsentierbare Eigenschaften. Wir halten die Klassifikationen jeweils für täuschend, weil der Inhalt der Klassifikation - als was die Gegenstände in der Wahrnehmung klassifiziert werden - von dieser Klassifikationsbasis abweicht und alltägliche reichhaltige Kategorien umfasst.

Proximale Reize

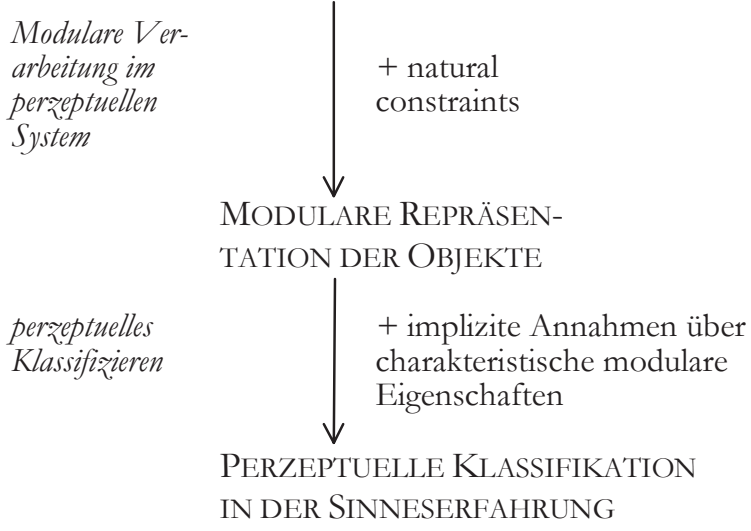

Abb. 7.2

Wie schon erwähnt, soll aber in diesem Abschnitt die Frage ausgeklammert werden, inwiefern der Besitz der Begriffe, als unter die fallend klassifiziert wird, theorieabhängig oder theorieunabhängig ist. Klassifikationsfähigkeiten sind daher nur darüber bestimmt, welche Zuordnung lokaler Objekte zu einer Äquivalenzklasse sie bewirken. Es handelt sich insofern nur um Fähigkeiten, lokale Objekte als gleich oder verschieden zu repräsentieren. Die Kategorie F, als zu der gehörend klassifiziert wird, ist als solche unbestimmt. Es ist aber wichtig festzuhalten, dass diese Kategorie von der Reihe der Eigenschaften $\mathrm{E}_{1}$ bis $\mathrm{E}_{\mathrm{n}}$, auf deren Basis klassifiziert wird, verschieden sein kann.

Was spricht nun für dieses Bild und allgemeiner für die Theorieunabhängigkeit perzeptueller Klassifikationsfähigkeiten? Hier lassen sich die Umstände anführen, unter denen man Klassifikationsfähigkeiten erwerben kann. 


\section{c) Der theorieunabbängige Erwerb klassifikatorischer Fäbigkeiten}

Angenommen, ich sehe zum ersten Mal eine Bachstelze. Ich beobachte, wie der Vogel an einem Teich hin und her hüpft, wie er mit dem Schwanz wippt, kurz aufflattert und auf einen Stein, einen Ast, ins Gras springt. Schließlich fliegt er davon. Es ist wahrscheinlich, dass ich das nächste Mal, wenn ich eine Bachstelze sehe, sie nun auch als solche visuell erkenne: Ich sehe sie als einen Vogel derselben Art, die ich zum ersten Mal am Teich gesehen habe. Ich habe mit anderen Worten eine Fähigkeit zum Klassifizieren von Bachstelzen als zu dieser Art gehörend erworben. Ganz ähnlich kann es mir mit Litschi ergehen. Wenn ich zum ersten Mal einige Litschi sehe, sie in die Hand nehme, sie schäle und esse, ist es wahrscheinlich, dass ich beim nächsten Sehen von Litschi sie unmittelbar als Früchte derselben Sorte erkenne.

Man kann offenbar perzeptuelle Fähigkeiten zum Erkennen von Arten oft sehr leicht erwerben, indem man einfach einige (oder ein einziges) Exemplar der Art eine Zeit lang mit einiger Ausführlichkeit beobachtet oder damit hantiert. Man braucht demnach keine Theorie darüber, was man eigentlich wahrnimmt, der Erwerb klassifikatorischer Fähigkeiten ist nicht schwach theorieabhängig.

Auch eine falsche Theorie über die wahrgenommenen Objekte verhindert den Erwerb der Fähigkeit nicht. Angenommen, ich habe vage davon gehört, dass es eine neue Art von Süßigkeiten gibt. In dem Moment, in dem ich zum ersten Mal eine Litschi sehe, schließe ich leichtfertig, dass das da vor mir diese Süßigkeit ist, die aus einer Mischung aus Zucker, Wasser, Gelatine und künstlichen Aromen produziert wird. Dennoch kann ich auf die beschriebene Weise eine Fähigkeit zum Klassifizieren von Litschi erwerben. Ich werde in unserer gewöhnlichen Umgebung, in der alle Litschi und nur Litschi wie Litschi aussehen, alle Litschi als gleich und als verschieden von anderen Früchten und von Süßigkeiten klassifizieren. Eine Theorie über das Wahrgenommene braucht daher keinen Einfluss auf die erworbene Klassifikationsfähigkeit auszuüben.

Demnach ist es auch zu erwarten, dass man vor verschiedenen theoretischen Hintergründen gleiche Klassifikationsfähigkeiten erwirbt. Angenommen, Paul glaubt, dass Litschi und Gummibärchen ihrem Wesen nach von gleicher Art sind. Dennoch muss er für die beiden Ausprägungen der Art unabhängige Klassifikationsfähigkeiten erwerben. Wenn er bisher die LitschiVarianten noch nie gesehen hat, kann er sie auch nicht erkennen, auch wenn er Gummibärchen als solche erkennt.

Paula glaubt dagegen, dass die Dinge, die wie Litschi aussehen, tatsächlich zwei verschiedenen Arten zugehören. Es soll demnach chinesische Litschi geben, die als Früchte am Baum wachsen, und deutsche, die auf den Produk- 
tionsbändern der Lebensmittelindustrie entstehen. Wenn sie zum ersten Mal Litschi sieht, kann sie aber nur die Klassifikationsfähigkeit erwerben, die alle Litschi als gleich oder verschieden klassifiziert - unabhängig davon, ob sie die Musterexemplare für chinesische oder deutsche Litschi hält. Angenommen, sie hält die Musterexemplare für deutsch. Dann werden ihr alle anderen Litschi visuell als von gleicher Nationalität vorkommen. Obwohl Paul und Paula daher ganz unterschiedliche Auffassungen von den Objekten und insbesondere dem Ausmaß haben, in dem sie gleich und verschieden sind, können sie doch nur bestimmte Klassifikationsfähigkeiten erwerben - eben diejenigen, die man auch ohne Theorie erwirbt. Der Erwerb dieser Fähigkeiten ist demnach auch nicht stark theorieabhängig.

Dieser Befund zu den Möglichkeiten des Erwerbs klassifikatorischer Fähigkeiten steht im Gegensatz zur Lernweise, die Kuhn beschreibt. ${ }^{28}$ Kuhn zufolge kann man Klassifikationsfähigkeiten nur erwerben, wenn die eigenen Klassifikationsversuche durch eine theoretisch geprägte Gemeinschaft kontrolliert und korrigiert werden. Demnach hängt es von diesem theoretischen Hintergrund ab, sowohl ob man eine solche Fähigkeit, als auch welche Fähigkeit man erwirbt. Die gegebenen Beispiele zeigen aber, dass beides falsch ist. Vielmehr können wir über theorieunabhängige Klassifikationsfähigkeiten für beobachterfreundliche Eigenschaften, Arten und Individuen verfügen, die in unserer gewöhnlichen Umgebung zuverlässig sind. Hierfür gibt es weitere Belege.

\section{d) Weitere Belege für die Theorieunabhängigkeit}

Wenn Klassifikationsfähigkeiten für einen beobachterfreundlichen Bereich nicht schwach theorieabhängig sind, setzt der Besitz dieser Fähigkeiten keine Theorie über den Bereich voraus. Es ist dann zu erwarten, dass Gemeinschaften, die keine Theorie über den Bereich haben, ihn dennoch zuverlässig richtig unterteilen - und damit weitgehend so unterteilen, wie es wissenschaftliche Gemeinschaften tun, die theoretisch tragfähige Unterscheidungen treffen. Die vorwissenschaftliche und die wissenschaftliche Taxonomie sollten weitgehend übereinkommen.

Einen besonders bemerkenswerten Beleg hierfür liefert die anthropologische Forschung zur Alltagsbiologie, d.h. zu den im Alltag verbreiteten Annahmen und Kategorien der biologischen Welt. Brent Berlin hat die alltagsbiologischen Klassifikationen der Tzeltal, einer Gruppe von Maya-Indianern, mit denen der wissenschaftlichen Systematik der Biologie verglichen. ${ }^{29}$

\footnotetext{
28 Siehe Kuhn (1974) und Kap. 3, Abschn. 4b(iii).

29 Siehe Berlin (1973); vgl. auch Berlin (1999).
} 
Insbesondere auf der alltagsbiologischen Ebene der ,generischen Arten', die in ihrer Allgemeinheit ungefähr der wissenschaftlichen Ebene der Spezies entspricht, findet sich eine weitgehende Übereinstimmung zwischen wissenschaftlichen und alltäglichen Klassifikationen. Von 471 generischen Pflanzenarten, die bei den Tzeltal allgemein bekannt sind, entsprechen 291 direkt wissenschaftlichen Spezies, soweit sie in der Umgebung der Tzeltal vorkommen. 98 weitere Tzeltal-Arten korrespondieren zwar mehreren wissenschaftlichen Spezies, die aber jeweils zur selben wissenschaftlichen Gattung gehören. In diesen Fällen entspricht die Alltagsart daher einer wissenschaftlichen Kategorie, die Wissenschaft unterteilt Spezies hier aber feiner. Nur in 82 Fällen entspricht der Alltagsklassifikation keine lokalisierte wissenschaftliche Kategorie, entweder weil im Alltag feiner unterschieden wird oder weil wissenschaftlich gesehen disparate Pflanzen in einer Kategorie zusammengefasst werden. In über $80 \%$ der Fälle stimmt daher die alltägliche Kategorisierung mit der wissenschaftlichen Systematik überein. Die wissenschaftliche Systematik wird aber vor dem Hintergrund einer ausgefeilten biologischen Theorie und Methodologie zur Entstehung, Identität und Verwandtschaft von Arten entwickelt. Ein solcher Hintergrund fehlt der Alltagsbiologie völlig. Die weitgehende Übereinstimmung zeigt, dass wir schon vorwissenschaftlich über Klassifikationsfähigkeiten verfügen, die sich auch nach wissenschaftlichen Standards in weiten Teilen als zuverlässig erweisen.

Dann können diese Klassifikationsfähigkeiten aber auch für schon entwickelte Wissenschaften epistemisch bedeutsam sein. Denn wenn sie vor verschiedenen theoretischen Hintergründen gleichermaßen verfügbar sind, können sie zur empirischen Entscheidung zwischen konkurrierenden Theorien beitragen. In der Wissenschaftsgeschichte gibt es vielfältige Belege für eine solche Konstanz von Klassifikationsfähigkeiten über theoretische Unterschiede hinweg. So ist es offensichtlich, dass die Identifikation von Himmelskörpern wie dem Mond oder der Sonne vor völlig verschiedenen theoretischen Hintergründen einmütig vorgenommen wird. Die theoretischen Auffassungen darüber, worum es sich jeweils handelt - etwa Planet oder Satellit -, haben keinen Einfluss auf die Fähigkeit, den Mond in der Wahrnehmung als solchen zu erkennen und als denjenigen zu identifizieren, den man zu anderen Gelegenheiten schon gesehen hat. Ganz ähnliche Konstanzen finden sich in vielen anderen Bereichen, etwa bei der Klassifikation von Gesteinsarten in der Geologie. So gab es zwar lange Zeit grundlegende Meinungsverschiedenheiten über die Entstehungsweise und das Alter etwa des Gneis. Werner hielt ihn für das zweitälteste, aus dem Urozean auskristallisierte Mineral. Lyell fasste ihn dagegen als metamorphes Gestein auf, das zu verschiedenen erdgeschichtlichen Perioden unter Hitze und Druck, aber ohne 
vollständiges Schmelzen entstanden sein konnte. Dabei war es aber unstrittig, über welche Gesteinsart man verschiedener theoretischer Auffassung war. Offenbar gab es keine theoriebedingten Schwierigkeiten, Gneis als solchen zu identifizieren.

In der Chemie schließlich zeigt sich im Umfeld der chemischen Revolution die Konstanz klassifikatorischer Fähigkeiten. Kuhn betont die Diskontinuität, wenn er schreibt:

Lavoisier [musste] ... seine Anschauung von vielen ... [vertrauten] Stoffen ändern. Er musste beispielsweise ein zusammengesetztes Erz sehen, wo Priestley und seine Zeitgenossen eine elementare Erde gesehen hatten. (Kuhn 1962, 130)

Aber Kuhn übergeht hier die Kontinuität der Klassifikationsfähigkeiten über theoretische Differenzen und mögliche begriffliche Veränderungen hinweg. Obwohl es sehr unterschiedliche Auffassungen etwa über die Natur von metallischem Quecksilber und mercurius calcinatus, dem roten Quecksilberoxid, gab, brauchte die Identifikation dieser Substanzen nicht problematisch zu sein. Man kann wissen, wie mercurius calcinatus und metallisches Quecksilber üblicherweise aussehen - man kann über lokal verlässliche Klassifikationsfähigkeiten für die Substanzen verfügen - unabhängig davon, welcher Theorie man anhängt. Damit kann man aber beispielsweise theorieunabhängig beobachten, dass sich mercurius calcinatus beim Erhitzen in metallisches Quecksilber umwandelt, ohne dass man Holzkohle zugeben muss. Dass diese Reaktion möglich ist, brachte die Phlogistontheorie aber in erhebliche Bedrängnis. Zuverlässige Klassifikationsfähigkeiten ermöglichen auf solche Weise theorieunabhängige empirische Befunde, die für konkurrierende Theorien evidenziell höchst bedeutsam sind..$^{30}$

In allen geschilderten Fällen theorieunabhängiger Klassifikationsfähigkeiten scheint es sich um Arten, Eigenschaften oder Individuen zu handeln, die vermutlich im oben bestimmten Sinn beobachterfreundlich sind. Dies spricht dafür, dass die Theorieunabhängigkeit auf dem dargestellten Zusammenspiel von modularer Reizverarbeitung und empirischem Erwerb impliziten klassifikatorischen Wissens beruht. Demnach ist das Wissen, das in solche Klassifikationsfähigkeiten einfließt, weder theoretisch noch theorieabhängig. Aber auch wenn man die modulare Theorie des Sehens nicht voraussetzt und damit das detaillierte Bild nicht teilt, zeigen die angeführten Befunde, dass viele Klassifikationsfähigkeiten und damit die in den unmittelbaren Erfahrungen enthaltenen Klassifikationen gemäß alltäglichen und wissenschaftlichen Kategorien weitreichend theorieunabhängig sind.

30 Vgl. auch meine kritische Einschätzung anderer Kuhn'scher Fälle aus der Wissenschaftsgeschichte in Kap. 3, Abschn. 4b(iv). 


\section{Zur Semantik von Beobachtungsausdrücken}

Perzeptuelle Klassifikationsfähigkeiten wurden bisher im Sinne der Fähigkeiten verstanden, Objekte als gleich oder verschieden zu repräsentieren. Allerdings werden in der Wahrnehmung Objekte auch als von bestimmter Art (als Rotkehlchen), mit bestimmten Eigenschaften (als herzförmig) oder von bestimmter Identität (als die Sonne) klassifiziert. Die Objekte werden so begrifflich bestimmt. Dieser begriffliche Inhalt von Wahrnehmungen kann oft durch einen entsprechenden Beobachtungssatz ausgedrückt werden. Es stellt sich die Frage, ob auch diese Inhalte theorieunabhängig sein können, oder ob man davon ausgehen muss, dass man nur vor einem geeigneten theoretischen Hintergrund über diese Begriffe verfügen kann oder Ausdrücke die entsprechende Bedeutung haben können.

Damit greife ich die Überlegungen auf, die in Kapitel 4 zur semantischen Theorieabhängigkeit diskutiert wurden. Feyerabend und Churchland begründen die Theorieabhängigkeit von Beobachtungen insbesondere damit, dass ihrer Ansicht nach eine Netzwerk-Semantik die plausibelste semantische Theorie für Beobachtungsausdrücke ist. In Kapitel 4 wurde nicht nur gezeigt, dass diese Annahme fatale Konsequenzen für die Objektivität von Beobachtungen hat. Es wurde auch klar, dass Feyerabends und Churchlands Argumentation für die Netzwerk-Semantik von Beobachtungsausdrücken sehr schwach ist. Ihr erstes Hauptargument, das Argument von der Semantik theoretischer Ausdrücke (siehe Kap. 4, Abschn. 3b), krankt daran, dass es nicht zwingend ist anzunehmen, dass die Bedeutung aller wissenschaftlichen Ausdrücke auf dieselbe Weise festgelegt wird. Auch wenn man akzeptiert, dass eine Netzwerk-Semantik für viele theoretische Ausdrücke angemessen ist, können Feyerabend und Churchland nicht überzeugend darlegen, dass dann auch Beobachtungsausdrücke ihre Bedeutung durch ein Netzwerk theoretischer Annahmen erhalten müssen. Im zweiten zentralen Argument, dem Ausschlussargument (Kap. 4, Abschn. 3c), werden zwei alternative semantische Konzeptionen - dass die Bedeutung durch Empfindungsqualitäten bzw. durch kausale Verknüpfungen mit Eigenschaften festgelegt wird -, zurückgewiesen. Aber auch wenn man dieser Zurückweisung folgt, bleibt das Argument anfällig gegenüber der Präsentation einer weiteren, alternativen semantischen Theorie für Beobachtungsausdrücke. Ich werde hier anknüpfen und argumentieren, dass man davon ausgehen muss, dass Beobachtungsausdrücke auf eine bisher unberücksichtigte Weise ihre Bedeutung bekommen können. Diese Weise der Festlegung der Inhalte ermöglicht es, dass eine Gemeinschaft unabhängig von einem theoretischen Hintergrund über Ausdrücke bzw. Begriffe für beobachterfreundliche Arten verfügt. 
Allerdings werde ich in diesem Abschnitt keine umfassende semantische Theorie für Beobachtungsausdrücke formulieren. Zum einen möchte ich hier nur argumentieren, dass eine Menge von Bedingungen hinreichend für Bedeutung bzw. Begriffsbesitz ist. Es ist damit nicht die Behauptung verbunden, dass damit die einzige mögliche Fundierung der Bedeutung von Beobachtungsausdrücken angegeben ist. Es kann durchaus weitere, ebenfalls hinreichende Faktoren für die Bedeutungsfestlegung geben. Zum anderen werde ich nicht anstreben, die Bedeutung von Ausdrücken auf eindeutig nicht-semantische Eigenschaften zurückzuführen. Vielmehr werden insbesondere Klassifikationsfähigkeiten und auch bestimmte Absichten oder Urteilsdispositionen zu den angegebenen Bedingungen gehören.

Beide Einschränkungen des Anspruchs sind aber mit dem Argumentationsziel vereinbar. Die Bedeutung eines Ausdrucks ist schon dann theorieunabhängig, wenn eine Gemeinschaft auch ohne theoretischen Hintergrund oder vor verschiedenen theoretischen Hintergründen über den Ausdruck mit dieser Bedeutung verfügen kann. Dies ist gegeben, wenn es theorieunabhängige Faktoren gibt, die zusammen hinreichend für die Festlegung der Bedeutung sind. Es ist in diesem Zusammenhang weder notwendig, dass diese Faktoren selbst aus einem bestimmten ontologischen, etwa naturalisierten Bereich, stammen, noch dass dies die einzigen Faktoren sind, die dem Ausdruck eine solche Bedeutung verschaffen können.

Bekanntermaßen haben insbesondere Saul Kripke und Hilary Putnam dafür argumentiert, dass auch solche Gemeinschaften über Ausdrücke insbesondere für natürliche Arten verfügen können, die noch keine oder nur radikal falsche wissenschaftliche Theorien über die Arten besitzen. ${ }^{31}$ Nach Kripke und Putnam kann die Bedeutung (Intension) für Art-Ausdrücke in zwei Schritten festgelegt werden. Zum ersten greift man auf irgendeine Weise ein Muster oder eine Menge von Mustern der Art heraus. Man zeigt beispielsweise auf ein Exemplar oder eine Reihe von Exemplaren oder benutzt eine Beschreibung, die auf einige Exemplare zutrifft. Zum zweiten wird durch eine objektive Ähnlichkeitsrelation zwischen den Exemplaren (oder den meisten Exemplaren) und anderen Gegenständen (auch in anderen möglichen Welten) eine Intension festgelegt. Im Fall von Gold könnte die Mustermenge beispielsweise in den Substanzen bestehen, die eine Gemeinschaft als ,Gold` bezeichnet. Die Intension umfasst dann alle Substanzen derselben Natur, etwa alle Atome derselben Ordnungszahl.

Es ist nun nahe liegend anzunehmen, dass ein solches Verfahren der Bedeutungsfestlegung für all diejenigen Arten, Eigenschaften und Individuen

31 Siehe Kripke (1980), Kap. III; Putnam (1975b). 
funktionieren kann, für die wir lokal zuverlässige Klassifikationsfähigkeiten besitzen. Diese Idee wurde insbesondere von Jessica Brown ausgearbeitet, und die folgenden Ausführungen greifen einige Merkmale dieser Ausarbeitung auf. ${ }^{32}$ Für die gegenwärtigen Belange sind insbesondere theorieunabhängige Klassifikationsfähigkeiten von Interesse, da dann auch die Fähigkeiten selbst kein theoretisches oder theorieabhängiges Wissen voraussetzen. Wie gezeigt, sind solche Fähigkeiten für beobachterfreundliche Arten möglich. Kann damit auch die Bedeutung von Beobachtungsausdrücken (der Besitz von Begriffen) festgelegt werden?

Angenommen, wir haben eine verlässliche, theorieunabhängige Klassifikationsfähigkeit für eine in unserer Umgebung beobachterfreundliche Art erworben, beispielsweise für Bachstelzen. Wir führen einen Ausdruck in unsere Sprache ein - etwa „Teichhopser“ -, womit wir die Art, als zu der gehörend wir hier klassifizieren, bezeichnen wollen. Doch dann ist es zu erwarten, dass dieser Ausdruck tatsächlich die Art der Bachstelzen bezeichnet. Denn zum einen haben wir eine lokal verlässliche Fähigkeit, ziemlich genau die lokalen Exemplare der Art dem Ausdruck zuzuordnen, und wir gebrauchen den Ausdruck in Übereinstimmung mit dieser Klassifikation. Zum anderen ist die Zugehörigkeit zu dieser Art gerade dasjenige, was diese Exemplare zentral gemeinsam haben.

Es ist daher zu erwarten, dass „Teichhopser“ erstens genau die Bachstelzen in unserer Umgebung bezeichnet. Auch einige wenige Bachstelzen in unserer Umgebung, die nicht Art-typisch springen, etwa weil sie einbeinig sind, und die unsere Klassifikationsfähigkeit daher nicht erfasst, werden demnach von „Teichhopser“ bezeichnet. Die wenigen Bachstelzen-Roboter, die aus dem fernen Land stammen, wo es sie als Teil eines Gartenteich-Bausatzes im Baumarkt zu kaufen gibt, fallen dagegen nicht in die Extension. Denn obwohl die lokalen Bachstelzen-Roboter zur Mustermenge gehören, die einbeinigen Bachstelzen aber nicht, sorgt die Bedingung der Artgleichheit mit der weit überwiegenden Mehrzahl der Musterexemplare dafür, dass die einen aus der Extension ausgeschlossen, die anderen dagegen hereingenommen werden. In gleicher Weise ist es zweitens zu erwarten, dass auch die Bachstelzen anderer, entfernter oder bloß möglicher Umgebungen von „Teichhopser“ bezeichnet werden.

Man könnte einige Einwände dagegen vorbringen, dass „Teichhopser“ auf die beschriebene Weise theorieunabhängig als Artbezeichnung eingeführt

32 Siehe J. Brown (1998); Grundzüge der Idee finden sich schon bei Evans (1982), 382/383. Während aber Brown Klassifikationsfähigkeiten für notwendig hält, damit Bedeutung auf insgsamt Putnam'sche Weise festgelegt werden kann, will ich nur plausibel machen, dass sie hinreichen. 
werden kann. Erstens könnte man einwenden, dass durch die Art der Einführung gar nicht klar ist, was genau der Ausdruck bezeichnet. Es ist demnach fraglich, ob man einen Ausdruck für die Art der Bachstelzen erhält oder aber einen Ausdruck, der auf alles zutrifft, was wie die lokalen, klassifizierten Objekte aussieht. Die eingesetzte Klassifikationsfähigkeit operiert auf der Basis einer Reihe $\mathrm{E}_{1}$ bis $\mathrm{E}_{\mathrm{n}}$ von theorieunabhängig repräsentierbaren Eigenschaften. Der alternativen Bedeutung zufolge bezeichnet unser eingeführter Ausdruck gerade alle diejenigen Objekte, die diese Eigenschaften besitzen. Demnach fallen alle Bachstelzen-Roboter in die Extension des Ausdrucks, die einbeinigen Bachstelzen dagegen nicht.

Brown argumentiert hier, dass sich solche Ausdrücke dann eindeutig die Art und nicht das Aussehen (bzw. eine Reihe modular repräsentierbarer Eigenschaften) bezeichnen, wenn die Benutzer des Ausdrucks sich klar darüber sind, dass das Fallen unter den Ausdruck nicht am Aussehen, sondern an den grundlegenden Eigenschaften der Objekte liegt. ${ }^{33}$ Diese Annahme brauchen die Sprachbenutzer nicht explizit zu machen. Es soll ausreichen, dass sie bereit sind, ihre Urteile im Lichte von Informationen über die grundlegenden Eigenschaften zu revidieren. Auf unsere Fälle übertragen müssen Sprachbenutzer etwa eine Klassifikation eines Roboters als Teichhopser auf die Information hin als irrig zurücknehmen, dass dessen grundlegende Eigenschaften ganz andere sind als bei den weitaus meisten anderen klassifizierten Teichhopsern. ${ }^{34}$

Diese Anforderung ist aber plausiblerweise oft erfüllt. Auch wenn man einen wissenschaftlichen Ausdruck über eine Klassifikationsfähigkeit einführt, beabsichtigt man häufig nicht, damit die Klasse der Objekte mit den Eigenschaften der Klassifikationsbasis $E_{1}$ bis $E_{n}$ zu bezeichnen, sondern eine wissenschaftlich interessante Art oder Eigenschaft. Wissenschaftlich von Interesse sind aber Kategorien, über die gesetzesartige Verallgemeinerungen möglich sind, die in Erklärungen oder induktive Schlüsse eingehen können. Wenn man erfährt, dass ein zunächst als $F$ klassifiziertes Objekt nicht in solcher Weise zu den weitaus meisten anderen, als Fs klassifizierten Dingen passt, ist es wissenschaftlich nur vernünftig, das Objekt nicht für ein $F$ zu halten. Dann bezeichnet $F$ aber nicht die Klassifikationsbasis, sondern eine davon verschiedene, wissenschaftlich interessante Art oder Eigenschaft.

Doch diese Antwort auf den ersten Einwand legt einen zweiten Einwand bzw. eine kritische Frage nahe. Setzt das Verständnis dafür, dass die eingeführten Ausdrücke wissenschaftlich interessante Eigenschaften und Arten

33 Siehe Brown (1998), 286/287.

34 Analoges gilt für Individuen. Vgl. Brown (1998), 287/288. 
bezeichnen, nicht theoretisches Wissen voraus? Müssen nicht die Sprecher etwa die grobe theoretische Kategorie kennen, der die Exemplare angehören sollen? ${ }^{35}$ Dem ist entgegenzuhalten, dass solches Wissen nicht notwendigerweise vorausgesetzt ist.

Dies zeigt sich daran, dass es plausibel erscheint, dass eine eindeutige Extension in lokalen, entfernten und bloß möglichen Umgebungen auch dann herausgegriffen werden kann, wenn sich die Sprachgemeinschaft über die grundlegende Natur der Objekte irrt. Angenommen, die Teichhopser sind entgegen den Annahmen der Sprachgemeinschaft tatsächlich keine Vögel, sondern gefiederte Säugetiere. Dies ändert nichts daran, dass der über die Klassifikationsfähigkeiten eingeführte Ausdruck eben diese Art von Säugetieren bezeichnet. Wenn die Sprecher von diesem grundlegenden Merkmal erfahren, werden sie nicht zum Schluss kommen, dass es Teichhopser gar nicht gibt - dass die vielen bisher als Teichhopser klassifizierten Tiere gar keine sind -, sondern dass Teichhopser von einer anderen allgemeinen Art sind als angenommen. Die Referenz des Ausdrucks bleibt daher von den theoretischen Annahmen über die grobe Beschaffenheit der Objekte unabhängig, solange damit nur eine wissenschaftlich interessante Klasse herausgegriffen wird.

Im Übrigen stellt sich für die Position, die im Einwand vorausgesetzt wird, ein gravierendes Problem. Wenn man davon ausgeht, dass die grobe theoretische Bestimmung einer Kategorie für die Festlegung der Referenz mitverantwortlich ist, wird unerklärlich, wie die Referenz einer sehr großen Anzahl von Eigenschafts- und Artbezeichnungen über viele Jahrhunderte offenbar stabil sein konnte, obwohl sich die theoretischen Annahmen bezüglich ihrer groben Beschaffenheit zum Teil radikal geändert haben. Eine Unmenge von Tierund Pflanzenbezeichnungen haben die Darwin'sche Revolution ohne gravierende Änderungen des Bezugs überstanden. Wechselnde geologische und mineralogische Theorien haben vielen Bezeichnungen für Gesteinsarten keine Referenzänderung gebracht. Und in der Chemie werden viele Trivialnamen oft ebenfalls schon sehr lange stabil über Änderungen theoretischer Auffassungen hinweg verwendet. Diese Stabilität erscheint für eine Semantik, die Referenz auf theoretische Annahmen gründet, unerklärlich. Vor dem Hintergrund der vorgeschlagenen Möglichkeit der Bedeutungsfestlegung ist hingegen eine solche Stabilität in all jenen Fällen, in denen Arten, Eigenschaften oder Individuen beobachterfreundlich sind, gerade zu erwarten. ${ }^{36}$

35 Dies ist Hansons Auffassung. Siehe Kap. 3, Abschn. 4b(i).

36 Auch Fälle, in denen eine leicht beobachtbare Klasse obsolet geworden ist - wie etwa „Hexe' (siehe Kap. 4, Abschn. 3c) -, sind durch die vorgeschlagene Semantik leicht zu erklären. In diesen Fällen hat sich herausgestellt, dass die als gleich klassifizierten Ob- 
Zudem schließt die vorgeschlagene Position eine semantische Rolle für theoretische Annahmen nicht aus. Verlässliche Klassifikationsfähigkeiten und objektives wissenschaftliches Interesse sind demnach zwar oft hinreichend dafür, dass eine Gemeinschaft über Ausdrücke für Arten, Eigenschaften und Individuen verfügt bzw. Begriffe für sie besitzt. Es ist jedoch denkbar, dass die Bedeutung der Ausdrücke überdeterminiert ist, so dass allein durch allgemeine theoretische Annahmen die Bedeutung ebenso festliegen würde wie durch Klassifikationsfähigkeiten. Aber eine solche theoretische KoFestlegung tut der Theorieunabhängigkeit der Bedeutung keinen Abbruch. Denn auch eine Gemeinschaft ohne theoretischen Hintergrund oder mit einem anderen Hintergrund könnte aufgrund der empirischen Bedeutungsfestlegung über Ausdrücke weitgehend derselben Bedeutung verfügen.

Damit wird erklärbar, wodurch die Bedeutung des Temperaturvokabulars bei den Freunden des Wärmestoffs festgelegt wird. ${ }^{37}$ Diese Churchland'sche fiktive Gemeinschaft gebraucht das Vokabular der Wärmestofftheorie, etwa den Ausdruck „hat einen hohen Wärmestoffdruck“, in unmittelbarer Reaktion auf Temperaturempfindungen. Churchland zufolge wird die Bedeutung dieser Wahrnehmungsurteile durch eine alltäglich gewordene Wärmestofftheorie festgelegt. Wie auch Churchland zugesteht, ist zu erwarten, dass die Freunde des Wärmestoffs sich wenig beeindruckt von der Mitteilung zeigen, dass es Wärmestoff gar nicht gibt. ${ }^{38}$ Sie werden ihre Wahrnehmungsurteile weiterhin für zutreffend halten. Während aber Churchland diese Reaktion als Beleg dafür wertet, wie schwierig es ist, tief im Alltag und in der Wahrnehmung verankerte theoretische Irrtümer einzusehen und zu korrigieren, liegt jetzt eine ganz andere Erklärung auf der Hand. Die WärmestoffFreunde verfügen über verlässliche Fähigkeiten, verschiedene Temperaturen perzeptuell zu unterscheiden und zu klassifizieren. Diese Fähigkeiten bestimmen die Bedeutung ihrer Beobachtungsurteile. Unsere Mitteilung besagt daher nur, dass semantisch unbedeutende, wenn auch weit verbreitete Annahmen über das so Bezeichnete falsch sind. Sie berührt nicht die Wahrheit der Beobachtungsurteile.

Insgesamt spricht somit Vieles dafür, dass - entgegen den in Kapitel 3 bis 5 diskutierten Gründen - direkte Wahrnehmungen vieler Eigenschaften, Arten und Individuen theorieunabhängig sind. Die Verarbeitung der Reize im

jekte tatsächlich durch keine wissenschaftlich interessante Gemeinsamkeit charakterisiert sind und insofern dem Ausdruck keine Referenz zukommt. Somit ist es der vorgeschlagenen Position zufolge semantisch möglich, dass Ausdrücke systematisch falsch angewendet werden.

37 Siehe Kap. 4, Abschn. 3b(iii).

38 Siehe Churchland (1979), 18-20. 
visuellen System ist aller Wahrscheinlichkeit nach modular; viele Arten, Eigenschaften und Individuen sind allein aufgrund der so repräsentierbaren Eigenschaften verlässlich klassifizierbar; und solche Klassifikationsfähigkeiten reichen für den Erwerb und den Besitz von entsprechenden Begriffen. Vor völlig verschiedenen theoretischen Hintergründen und auch völlig ohne einen solchen Hintergrund sind daher die gleichen perzeptuellen Klassifikationen und Wahrnehmungsurteile möglich.

Wie schon gesehen, kann auch die wissenschaftliche Einschätzung der Verlässlichkeit solcher Wahrnehmungen nicht zu einer weit greifenden Theorieabhängigkeit führen. (Siehe Kap. 5, Abschn. 4b.) Denn direkte Wahrnehmungen gelten als prima facie glaubwürdig. Eine Theorie kann daher nur Einfluss auf die Einschätzung einer Wahrnehmung ausüben, indem auf ihrer Grundlage die Wahrnehmung als unzuverlässig eingeschätzt wird. Damit setzt sie sich aber in Widerspruch zu einem prima facie akzeptablen empirischen Befund. Eine Theorie, die insgesamt optimal empirisch bestätigt sein soll, kann keine große Anzahl solcher Befunde als unzuverlässig erklären.

\section{Wahrnehmungen mit Hilfe von Instrumenten}

Neben direkten Wahrnehmungen zählen auch Beobachtungen mit Hilfe von Wahrnehmungsinstrumenten wie dem Lichtmikroskop und mit bildgebenden Verfahren wie der Computertomographie zu den perzeptuellen Beobachtungen. Der Grund hierfür ist, dass in allen Fällen charakteristische perzeptuelle Erkennensfähigkeiten dafür eingesetzt werden, um im Beobachtungsprozess zu einer ersten begrifflichen Repräsentation zu kommen. (Siehe Kap. 6, Abschn. 2a.)

Es ist sinnvoll, für die Frage der Theorieabhängigkeit zwischen Beobachtungen mit Wahrnehmungsinstrumenten und mit bildgebenden Verfahren zu unterscheiden. Für die Unterscheidung kann man sich an zwei Merkmalen orientieren, wobei eine Reihe von Grenzfällen bleibt. Zum ersten nimmt man mit Hilfe eines Wahrnehmungsinstruments typischerweise primär wissenschaftliche Gegenstände und deren Eigenschaften wahr, bei bildgebenden Verfahren dagegen Bilder. Wenn man durch ein gewöhnliches Lichtmikroskop schaut, kann man damit beispielsweise ein Pantoffeltierchen sehen. Mit einem bildgebenden Verfahren wie der Computertomographie kann man dagegen beispielsweise einen Tumor auf einem Bild sehen. Etwas auf einem Bild zu sehen impliziert aber nicht, das Ding selbst - etwa mit Hilfe des Bildes zu sehen. Ich kann eine Person auf einem Bild wahrnehmen, auch wenn sie schon gestorben ist und sie daher nicht wahrnehmbar ist. Bei Wahrnehmungsinstrumenten sieht man oft die wissenschaftlichen Gegenstände quasi ,durch das Instrument hindurch'. 
Zweitens ist bei bildgebenden Verfahren das Bild typischerweise Ergebnis einer digitalen Datenverarbeitung. In bildgebenden Verfahren werden oft Informationen über einzelne Punkte des abgebildeten Bereichs separat gewonnen und als solche symbolisch repräsentiert, diese Repräsentationen werden per Computer verarbeitet und die Pixel schließlich zu einem Bild zusammengesetzt. Beispielsweise gewinnt man in der Computertomographie Informationen über die Röntgenabsorption einzelner Volumenteile des Körpers, indem dieser schichtweise aus vielen Richtungen durchstrahlt wird. Die so gewonnenen Daten werden dann per Computer aufbereitet und zu einem Bild zusammengesetzt. Demgegenüber findet bei Wahrnehmungsinstrumenten in der Regel keine digitale, auf einzelne Bildpunkte bezogene Verarbeitung symbolischer Repräsentationen statt; vielmehr werden die Signale als ganze und durch bloße physikalische Komponenten (etwa Linsen, analoge Verstärker, etc.) verarbeitet.

Wenn man so zwischen Wahrnehmungsinstrumenten und bildgebenden Verfahren unterscheidet, führt dies zu einigen Grenzfällen. Gewöhnliche Röntgengeräte funktionieren zwar durch analoge und bloß physikalische Signalverarbeitung, man stellt aber Bilder her und nimmt etwa einen Knochenbruch auf einem Foto oder einem Schirm wahr. Ganz ähnlich funktionieren Elektronenmikroskope. Aber die Theoriebeladenheit dieser Grenzfälle lässt sich durch die Diskussion der klaren Fälle mit behandeln.

Bei Wahrnehmungen mit Hilfe von Instrumenten wird zunächst ein Signal, das vom Untersuchungsgegenstand ausgeht oder bei ihm hervorgerufen wird, aufgefangen. Beispielsweise fällt das Licht, das von einer durchleuchteten Flüssigkeitsschicht ausgeht, in die Objektivlinse eines Mikroskops. Das Signal wird im Gerät verarbeitet, beispielsweise durch Linsen und Prismen gebeugt, und schließlich so aufbereitet, dass es wahrnehmbar wird. Wenn alles gut geht, kann ein Beobachter auf solche Weise Dinge, die sich in der Flüssigkeit befinden, wahrnehmen, sie beispielsweise als Pantoffeltierchen erkennen, und zu Wahrnehmungsurteilen gelangen.

Solche Beobachtungen sind weitgehend theorieunabhängig möglich. Die Gründe hierfür sind im Kern dieselben, die auch für die Theorieunabhängigkeit vieler direkter Wahrnehmungen sprechen. Erstens ist festzustellen, dass Arten, Eigenschaften oder Gegenstände relativ zu Wahrnehmungsinstrumenten beobachterfreundlich sein können. Das bedeutet hier, dass beispielsweise Exemplare einer Art durch eine Menge von Eigenschaften $\mathrm{E}_{1}$ bis $E_{n}$ ausgezeichnet sind, die mit Hilfe des Wahrnehmungsinstruments durch das visuelle System modular repräsentiert werden können, und etwas in der relevanten Umgebung diese Eigenschaften ziemlich genau dann besitzt, wenn es zu dieser Art gehört. Die Arten und Gegenstände vieler Bereiche sind 
solchermaßen Instrumente-beobachterfreundlich. Beispielsweise haben verschiedene Gattungen von Einzellern, die sich in Tümpeln finden - Pantoffeltierchen, Rädertierchen, Glockentierchen, Amöben etc. -, oft solche charakteristischen Eigenschaften, die mit Hilfe eines Lichtmikroskops modular visuell repräsentierbar sind.

Zweitens ist es dann auch möglich, theorieunabhängige Klassifikationsfähigkeiten für diese Arten zu erwerben. Man kann lernen, mit Hilfe eines Mikroskops Pantoffeltierchen als solche zu erkennen und von Rädertierchen zu unterscheiden, ohne dass man hierfür einen theoretischen Hintergrund benötigt. Abgesehen vom Umgang mit dem Lichtmikroskop läuft dieser Erwerb bei Einzellerarten des Tümpels nicht anders ab als bei den Vogelarten des Vorgartens. Konzentriertes Zuschauen, wenn einige Pantoffeltierchen durch das Blickfeld schwimmen, reicht oft aus, um die Pantoffeltierchen des nächsten Tümpels als von derselben Gattung perzeptuell klassifizieren zu können. Drittens schließlich spricht nichts dagegen, auch Begriffe für diese Arten durch solche Instrumente-Erkennensfähigkeiten externalistisch zu erwerben. Dies zeigt aber, dass solche Beobachtungen mit Hilfe von Instrumenten, was die perzeptuelle Klassifikation und die Semantik der Begriffe angeht, theorieunabhängig möglich sind.

Natürlich gestaltet sich nicht alles Beobachten und perzeptuelles Klassifizieren mit Instrumenten so einfach wie bislang beschrieben. Zum einen erfordert die Wahrnehmung mit einem Instrument in der Regel eine gewisse Übung im Umgang mit dem Gerät. Zum anderen sind die relevanten Merkmale, die eine Klassifikation ermöglichen, nicht immer so offensichtlich. Doch die Fähigkeiten, mit dem Gerät richtig umzugehen und die Aufmerksamkeit auf die wichtigen Merkmale zu richten, brauchen selbst nicht theorieabhängig zu sein, auch wenn sie oft im Rahmen einer disziplinären Ausbildung gelehrt werden. ${ }^{39}$

Allerdings kann die Einschätzung der Verlässlichkeit partiell theorieabhängig sein. Wie oben festgestellt, muss die Verlässlichkeit von Wahrnehmungen, die man mit Hilfe von Instrumenten macht, in der Regel erst begründet werden. (Siehe Kap. 5, Abschn. 3b.) Hierbei können theoretische Gründe für oder auch gegen die Verlässlichkeit sprechen.

Aber abgesehen von theoretischen Gründen kann man auch empirische Gründe für oder gegen die Verlässlichkeit anführen. Gerade bei perzeptuellen Beobachtungen können solche Begründungen besonders aussagekräftig sein. In Kapitel 5 wurden einige dieser Verfahren, auf die insbesondere Hacking und Franklin hingewiesen haben, schon ausführlich diskutiert. So kann man

39 Vgl. Hacking (1983), Kap. 10. 
die Verlässlichkeit von Wahrnehmungen mit vielen Instrumenten für einen Teilbereich durch direkte Wahrnehmungen derselben Objekte überprüfen. Darauf aufbauend schließt man dann auf die Verlässlichkeit des Instruments auch in Bereichen, die der direkten Wahrnehmung nicht zugänglich sind. Oder man stützt die Verlässlichkeit dadurch, dass ein erwarteter Befund sich einstellt, oder aber sich mit verschiedenartigen Instrumenten gleiche Wahrnehmungen machen lassen. Eine solche Koinzidenz liefert gerade bei perzeptuellen Beobachtungen eine besonders gute Begründung, da die Wahrnehmungsinhalte oft sehr reichhaltig sind, etwa viele strukturelle Details der Objekte aufzeigen. Wenn verschiedene Verfahren solchermaßen inhaltsreich zu gleichen Ergebnissen führen, stützt dies die innerwissenschaftliche Annahme ihrer Verlässlichkeit besonders gut. Diese Stützung ist aber weitgehend theorieunabhängig.

Schließlich lässt sich mit gezielten Eingriffen in den Beobachtungsprozess und auf das Objekt die Genese des Beobachtungsergebnisses kausal analysieren. So lassen sich beispielsweise Merkmale der Ergebnisses, die sich bei Eingriffen auf das Objekt und durch das Austauschen des Objekts verändern, als dessen Beiträge identifizieren. Was dagegen durch Eingriffe in den Abbildungsprozess veränderbar ist, kann unter geeigneten Umständen als dessen Beitrag betrachtet werden. So gewinnt man theorieunabhängige Gründe dafür, den Beobachtungsprozess hinsichtlich bestimmter, damit wahrnehmbarer Eigenschaften für verlässlich zu halten. ${ }^{40}$

Insgesamt gilt, dass sich für oder gegen die Verlässlichkeit von Instrumentewahrnehmungen häufig vielfältige empirische, nicht weiter theorieabhängige Gründe anführen lassen. Das hat aber Konsequenzen für die Theorieabhängigkeit der Einschätzung insgesamt. Eine Theorie kann Beobachtungen, die empirisch für zuverlässig eingeschätzt wurden, nur zu Lasten ihrer eigenen empirischen Bestätigung für unzuverlässig erklären. Denn sie widerspricht damit den empirischen Befunden, welche die positive Einschätzung der Beobachtungen stützen. Gleiches gilt für Theorien, die solche Beobachtungen für zuverlässig erklären, die empirisch für unzuverlässig gehalten werden. Eine Theorie kann daher nicht in großem Umfang den empirisch getroffenen Verlässlichkeitseinschätzungen für Instrumentewahrnehmungen widersprechen, ohne selbst an empirischer Stützung einzubüßen. Von Theorien, die empirisch optimal erfolgreich sind, kann die Verlässlichkeitseinschätzung deshalb nur in einer Minderzahl der Fälle abhängen.

40 Siehe hierzu Kap. 5, Abschn. 3d. 


\section{Beobachtungen mit bildgebenden Verfahren}

Bei bildgebenden Verfahren werden üblicherweise mehrere Signale, die oft verschiedene Teile oder Teilvolumina des Untersuchungsobjekts betreffen, über eine gewisse Zeitspanne hinweg gesammelt. Diese Signale werden dann typischerweise elektronisch verarbeitet und schließlich zu einem Bild zusammengesetzt. Bei der Computertomographie sammelt man zunächst Informationen darüber, wie durchlässig etwa ein menschlicher Körper aus verschiedenen Richtungen für Röntgenstrahlen ist. Daraus berechnet man die Röntgenabsorption einzelner Volumenelemente, sogenannter Voxel. Diese Werte stellt man in Graustufen in Bildern, den Computertomogrammen, dar. Bei der Magnetresonanz-Tomographie wird ein Signal erzeugt, indem man an das Objekt zunächst ein starkes homogenes Magnetfeld anlegt. Hierdurch richten sich insbesondere die Protonen der Kerne mit ihrem Spin parallel zu diesem Magnetfeld aus. Dann überlagert man einen Puls eines oszillierenden Magnetfelds mit üblicherweise gerade derjenigen Frequenz, die der Energiedifferenz zwischen der parallelen Ausrichtung und einer Ausrichtung senkrecht zum homogenen Magnetfeld entspricht. Dadurch springen die Protonen in einen angeregten, antiparallelen Zustand. Nach Ende des Pulses fallen sie wieder in den parallelen Grundzustand zurück, wobei sie ein charakteristisches Magnetfeld emittieren. Dieses Magnetfeld und dessen räumliche und zeitliche Verteilung wird gemessen und daraus die Dichteverteilung der Protonen im Objekt errechnet.

In einer besonderen Variante, der sog. funktionalen MagnetresonanzTomographie, die in der Neuroforschung weit verbreitet ist, macht man sich oft den Umstand zunutze, dass das magnetische Moment der roten Blutkörperchen (genauer: des Hämoglobins) davon abhängt, ob sie Sauerstoff transportieren. ${ }^{41}$ Aufgrund dieser Abhängigkeit kann man eine zeitliche Veränderung des Sauerstoffgehalts des Bluts in einem Blutgefäß durch Magnetresonanz-Tomographie messen. Zudem geht man von den folgenden physiologischen Zusammenhängen aus. Wenn in einem Gehirnbereich die Aktivität der Neuronen zunimmt, steigt die Durchblutung der direkt versorgenden Blutgefäße. Die Neuronen entnehmen aber trotz erhöhter Aktivität dem Blut nicht mehr Sauerstoff. Damit steigt bei neuronaler Aktivität insgesamt der Sauerstoffgehalt des Blutes im aktiven Bereich. Daher geht man davon aus, dass Darstellungen der Veränderungen des magnetischen Signals des Hämoglobins eine veränderte neuronale Aktivität abbilden.

41 Für das Folgende siehe Turner/ Jezzard (1994), Cohen (1996) und Horwitz/ Friston/ Taylor (2000). 
Ist es nicht offensichtlich, dass Beobachtungen mit solchermaßen produzierten Bildern massiv theorieabhängig sind? Meiner Ansicht nach ist dies nicht so. Ich werde die verschiedenen Varianten der Theorieabhängigkeit einzeln durchgehen, zunächst die Frage, ob die Festlegung der Inhalte theorieabhängig ist.

\section{a) Inbalte}

Bei Beobachtungen mit bildgebenden Verfahren lassen sich zunächst Inhalte auf zwei Stufen unterscheiden. Zum einen haben die Bilder, zum anderen die Wahrnehmungen der Bilder Inhalte. Bei den Bildern, die durch solche Verfahren wie die Computertomographie zustande kommen, kann man zunächst fragen, was einzelne Pixel des Bildes (oder einzelne Voxel des dreidimensionalen Datensatzes) anzeigen. Hierbei ist man zunächst darauf verwiesen, was man über das Signal und dessen Verarbeitungsweise weiß. So stehen die Grauwerte bei einem Computertomogramm für das Maß, in dem ein Gewebeteil Röntgenstrahlen absorbiert. Dieses Maß der Absorption ist normiert und wird in Hounsfield-Einheiten angegeben, üblicherweise wird auf Computertomogrammen mit angezeigt, welche Grauwerte für wieviele Einheiten stehen. (Vgl. Abb. 7.3.) Das Bild funktioniert damit in dieser Hinsicht praktisch wie ein Messgerät mit sehr komplexer Anzeige. Es zeigt sehr viele Messwerte an, wobei die gemessenen Größen farblich unterschieden und räumliche Relationen zwischen den gemessenen Punkten wiedergegeben werden.

Es ist plausibel anzunehmen, dass diese Bildinhalte und ihre Verwendung oft theorieabhängig sind. Die Grauwerte einzelner Pixel stehen gemäß der explizit angegebenen Skala für bestimmte Messwerte. Um sie in Erfahrung zu bringen, muss man die Grauwerte mit der Skala abgleichen. So kann man ablesen, wofür sie stehen. Soweit die Inhalte der Pixel durch die Skala festgelegt werden, sind sie begrifflich, und man muss über diese Begriffe verfügen, um sie ablesen zu können. Oft besitzt man diese Begriffe - etwa den der Röntgenabsorption oder den des magnetischen Moments - aber nur, weil man über Theorien für diese Größen verfügt.

Allerdings könnte man hier - ähnlich wie schon bei der Diskussion von Messgeräten in Kapitel $4-42$ einwenden, dass tatsächlich die Inhalte der angezeigten Pixel nicht von der angegebenen Skala abhängen. Demnach stehen die Pixel vielmehr für diejenigen Größen, die sie tatsächlich verursacht haben, unabhängig davon, ob wir diese Verursachungsrelationen kennen oder

42 Siehe Kap. 4, Abschn. 2. 
über Begriffe für die Ursachen verfügen. Diese informationalen Inhalte der Pixel wären dann theorieunabhängig.

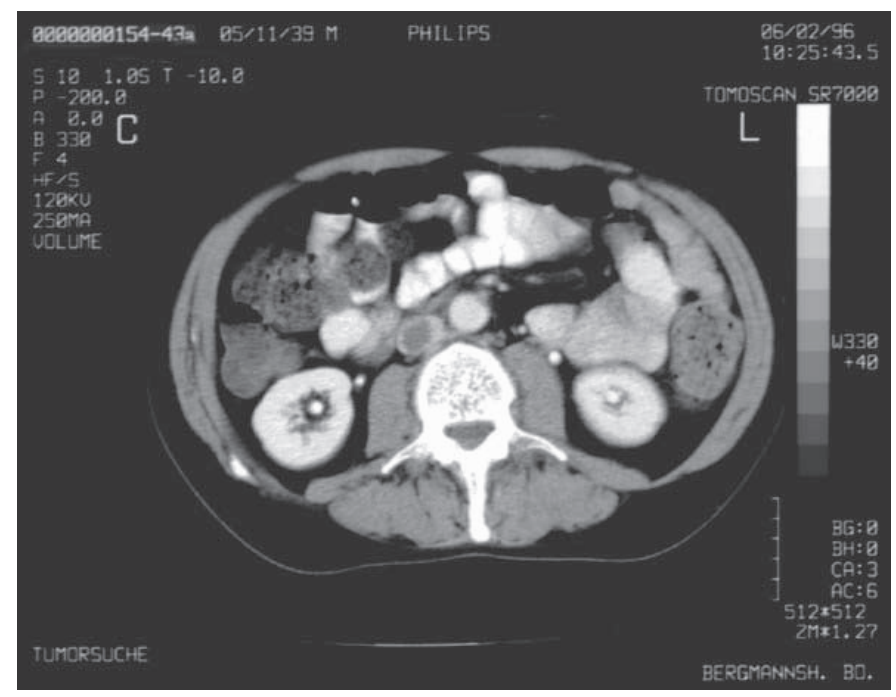

Abb. $7.3^{43}$

Diesem Einwand muss man aber zwei Punkte entgegenhalten. Zum ersten kommen die Pixelfarben bei bildgebenden Verfahren in der Regel durch eine ausdrückliche Kodierung zustande. Es werden zunächst Messwerte, etwa für die Röntgenabsorption eines Voxels, berechnet. Dann werden diese Messwerte für die Produktion des Bildes in Graustufen umgesetzt. Die Regeln für die Kodierung der Messwerte werden ins Programm, nach dem die Bilder erzeugt werden, hineingeschrieben. Es ist daher nicht zu bestreiten, dass die Pixel als solche - durch die Weise, wie sie produziert werden - die in der Kodierungsregel angenommenen Werte begrifflich repräsentieren.

Dies schließt zwar nicht aus, dass die Pixelwerte zusätzlich informationale Inhalte haben und ihre Ursachen begriffsunabhängig repräsentieren. Es ist zum zweiten zunächst aber unklar, wie darauf begriffsunabhängig zugegriffen werden soll. Die graue Farbe eines einzelnen Pixelwerts ist als solche genauso ,stumm ' wie der uninterpretierte Zeigerstand eines Messgeräts. Erst indem man den Graustufen Größen zuweist und damit interpretiert, werden sie wissenschaftlich verwendbar. Und diese Interpretation setzt gerade wieder Begriffe voraus.

43 Aus Bergmannsheil (1996). 
Allerdings gibt es auch wichtige Unterschiede zwischen gewöhnlichen Messgerätsanzeigen und den Bildern, die man mit bildgebenden Verfahren produziert. Denn anders als ein Zeigerstand kann ein Bild neben Inhalten der Pixel auch noch weitere Inhalte haben. So kann auf dem Bild insgesamt etwas abgebildet sein, das über die begrifflichen Inhalte, die in den Farbwerten der Pixel kodiert sind, hinausgeht und von diesen unabhängig ist. Diese Inhalte sind nichtbegrifflich, sie sind rein ,piktorial'. Dies lässt sich am Beispiel der Abbildung 7.3 zeigen. Es handelt sich hier um ein Computertomogramm, das einen Querschnitt des Bauches eines Menschen zeigt. Zum einen stehen die Grauwerte einzelner Pixel des Bildes für die Röntgenabsorption im Gewebe. Diese Kodierung wird durch die angezeigte Skala explizit gemacht. Zum anderen werden aber Knochen und Muskeln - unten in der Mitte etwa die Wirbelsäule und die Rückenmuskulatur - sowie verschiedene Organe abgebildet.

Damit Knochen, Muskeln und Organe so abgebildet werden, brauchen sie aber nicht gesondert in Grauwerten kodiert zu werden. Die Objekte tauchen automatisch auf dem Bild auf, indem man dieses aus Werten für die Röntgenabsorption zusammenbaut. Eine Gemeinschaft, die solche Computertomogramme erstellt, braucht daher nicht über Begriffe für die Objekte zu verfügen, um Bilder haben zu können, auf denen sie repräsentiert sind. Diese Inhalte der Abbildung sind daher insofern theorieunabhängig.

Ganz ähnlich stellt sich die Situation bei der funktionalen MagnetresonanzTomographie dar. Auch dort werden einerseits die Magnetsignale einzelner Teilvolumina explizit kodiert. Andererseits werden damit aktive Bereiche im Gehirn, deren Umfang, deren gleichzeitige oder aufeinanderfolgende Aktivität, vielleicht deren Koordination und die kausalen Abhängigkeiten zwischen ihnen abgebildet. Zwar hat man weitgehend explizites Wissen darüber, wie es zur Abbildung der Aktivität kommt. Man stützt sich unter anderem auf den oben dargestellten Zusammenhang zwischen neuronaler Aktivität und Sauerstoffkonzentration im Blut. Dieses Wissen setzt den Begriff etwa der neuronalen Aktivität voraus. Aber man braucht über solches Wissen nicht zu verfügen, damit die Aktivität auch abgebildet wird.

Obwohl man seit 1992 mit Hilfe der funktionalen Magnetresonanz-Tomographie die Gehirnaktivität beobachtet, ${ }^{44}$ weiß man erst seit 2001, dass das gemessene Signal weniger mit der Feuerungsrate als vielmehr mit der synaptischen Aktivität der Neuronen in einem Bereich zusammenhängt. Daher weiß man nun, dass auf den Bildern eher der Input und die interne Verarbeitungs-

44 Siehe Kwong et al. (1992) u. Ogawa et al. (1992). 
aktivität als der Output einer Neuronenpopulation abgebildet wird. ${ }^{45}$ Dieses Wissen ist sicherlich von Nutzen, wenn man daran geht, ein Bild zu interpretieren. Bei einer solchen Interpretation macht man sich oft den Inhalt der Bilder wissenschaftlich zunutze, indem man auf explizites Wissen über das Zustandekommen des Bildes zurückgreift. Aber auch unabhängig vom Wissen über das genaue Zustandekommen des Bildes kann dieses entsprechende Inhalte haben. Auch bevor man wusste, dass eher der Input als der Output einer Neuronenpopulation abgebildet wird, wurde auf den Magnetresonanz-Tomogrammen eben diese Form der Aktivität abgebildet. Es ist daher offenbar unnötig, den Zusammenhang zu kennen, um Bilder mit entsprechenden Inhalten zu haben. Zudem gehen die möglichen piktorialen Inhalte über die bloße Aktivität hinaus. Möglicherweise werden damit Eigenschaften von Komponenten des Geistes wie Modularität oder Vernetzung abgebildet. Wieder braucht man darüber nichts zu wissen, um davon Bilder zu erhalten - Bilder, mit denen man diese Komponenten aber dann entdecken könnte.

Ein Großteil der Inhalte von Bildern bildgebender Verfahren sind daher nichtbegrifflich. Man braucht demnach nicht für den Begriffsbesitz schon über Theorien zu verfügen, damit die Bilder überhaupt Inhalte haben. Vielmehr kann vieles abgebildet sein, das man noch nicht kennt und wofür man noch keine Begriffe besitzt. Zwar stellt eine wissensgestützte Interpretation der Bilder eine Möglichkeit dar, diese Inhalte wissenschaftlich verfügbar zu machen. Sie ist aber keinesfalls die einzige Möglichkeit. Die nichtbegrifflichen Inhalte der Bilder können auch über Wahrnehmungen rezipiert werden, die selbst theorieunabhängig sind. Um dies zu sehen, ist es hilfreich zu überlegen, wodurch der nichtbegriffliche Inhalt von Bildern festgelegt wird.

Es ist nicht leicht zu sagen, wodurch genau bestimmt wird, was auf Bildern, die mit einem bildgebenden Verfahren erzeugt werden, abgebildet ist. Allerdings scheint klar, dass die folgenden Bedingungen oft ausreichen. Angenommen erstens, verschiedene, beim Menschen vorkommende Organe führen mittels des Verfahrens zu unterscheidbaren Bildern. Das bedeutet, dass man perzeptuelle Klassifikationsfähigkeiten besitzen kann, die es erlauben, mit den Bildern die verschiedenen Organe recht zuverlässig zu unterscheiden. Zweitens sind diese Klassifikationsfähigkeiten ähnlicher Art wie diejenigen, mit denen man direkt oder mit Hilfe eines Wahrnehmungsinstruments die Organe unterscheiden könnte, falls sie so wahrnehmbar sind bzw. wären. Demnach sind es entweder dieselben Klassifikationsfähigkeiten, mit denen man etwa das Herz direkt - etwa bei einer Sezierung - erkennt; oder es

45 Siehe Logothetis et al. (2001); vgl. auch Horwitz/ Friston/ Taylor (2000), 838. 
sind Klassifikationsfähigkeiten, mit denen ein solches Erkennen möglich wäre, wenn die direkte oder instrumentelle Wahrnehmung möglich wäre; oder es sind hierzu recht ähnliche Klassifikationsfähigkeiten. Die Möglichkeit solchen Klassifizierens reicht in der Regel, um zu sagen, dass die Objekte und ihre Eigenschaften abgebildet sind.

Nehmen wir zur Illustration gewöhnliche Fotografien. Damit eine Person auf einem Foto abgebildet ist, reicht es aus, wenn gilt: Erstens führte das Fotografieren kausal - unter Mitwirkung der Person - zu einem Foto, auf dem die Person von anderen Personen auf dem Foto und anderen relevanten Personen gut unterscheidbar und als diese Person identifizierbar ist. Zweitens genügt hierzu eine Klassifikationsfähigkeit ganz ähnlich der Fähigkeit, mit der man die Person direkt als solche erkennt. Entweder braucht man bloß diese Fähigkeit oder vielleicht eine systematische Variante, die sich aus dieser Fähigkeit und der allgemeinen Fähigkeit, Dinge auf Fotografien zu erkennen, ergibt.

Wenn dies hinreicht, dann ist es erstens theorieunabhängig, welche Dinge und Eigenschaften auf dieser Grundlage auf Bildern abgebildet sind. Denn was derart abgebildet ist, hängt nicht von einem theoretischen Hintergrund, sondern nur von den objektiven Möglichkeiten des perzeptuellen Erkennens ab. Zweitens ermöglicht dies aber auch eine theorieunabhängige perzeptuelle Klassifikation. Wenn die wahrgenommenen Eigenschaften, auf deren Grundlage perzeptuell klassifiziert wird, modular repräsentierbar sind und die Objekte bezüglich der Abbildungsmethode beobachterfreundlich sind, sind auch die Klassifikationen theorieunabhängig. Es ist aber bei bildgebenden Verfahren ebenso plausibel wie bei direkten Wahrnehmungen und Instrumentewahrnehmungen, dass diese weiteren Bedingungen oft erfüllbar sind. Demnach können auch perzeptuelle Klassifikationen mit Hilfe von bildgebenden Verfahren theorieunabhängig sein.

Es ist von hier aus nur noch ein kleiner Schritt zu einem theorieunabhängigen Erwerb von Begriffen mit Hilfe von Bildern. Ein Beispiel hierfür ist der Begriff des Mesosoms. George Chapman und James Hillier, zwei Pioniere der Elektronenmikroskopie, haben als erste 1953 mit dem Elektronenmikroskop im Innern von Bakterien Objekte gefunden, die sie als periphere Körper bezeichneten. ${ }^{46}$ Anfang der 1960er Jahre häuften sich Befunde von meist großen, an die Zellmembran angeschlossenen Strukturen, oft in der Nähe der Bereiche, an denen sich die Bakterien gerade teilten. Philip Fitz-James nannte

46 Historisch und philosophisch wird der Fall von Rasmussen (1993), Culp (1994) und Hudson (1999) untersucht. Allerdings konzentrieren sich diese Studien auf die methodologische Frage, wie die Unzuverlässigkeit der Präparationsmethode festgestellt wurde, und nicht darauf, wie der Begriff des Mesosoms erworben wurde. 
sie Mesosome. Abbildung 7.4 zeigt ein solches Mesosom. Man hatte offenbar keine Schwierigkeiten, gut präparierte Exemplare dieser Strukturen auf elektronenmikroskopischen Aufnahmen als solche Mesosome zu klassifizieren. Man untersuchte die Entwicklung und Funktion von Mesosomen. Man ging davon aus, dass Mesosome unter anderem bei der Zellteilung an der Bildung der neuen Zellmembran und der Replikation der Chromosomen beteiligt sind. Die Grafik in Abbildung 7.5, die in ein Lehrbuch der Mikrobiologie der 1970er Jahre Eingang fand, zeigt, wie sich manche die Entwicklung vorstellten.

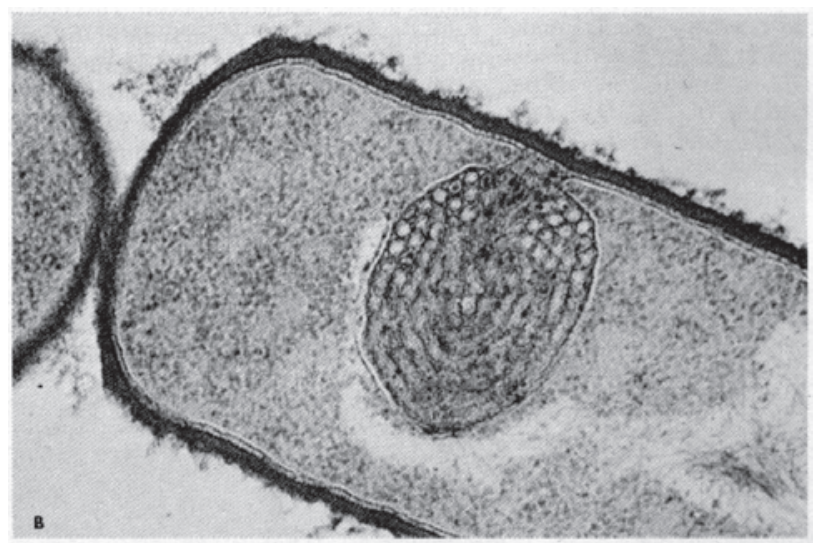

Abb. $7.4^{47}$

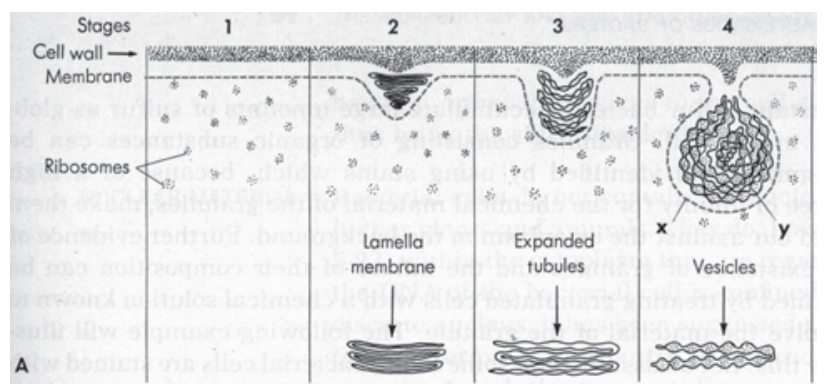

Abb. $7.5^{48}$

Im Laufe der 1970er und -80er Jahre kam man allerdings zur Überzeugung, dass Mesosome gar keine Zellorganellen sind, sondern lediglich Artefakte der

47 Aus Pelczar/ Reid/ Chan (1977), 95.

48 Aus Pelczar/ Reid/ Chan (1977), 95; ursprünglich aus Rogers (1970). 
Präparation der Bakterien darstellen. Es stellte sich heraus, dass die beobachtbare Morphologie der Mesosome stark von der Präparationsmethode abhing. Zudem wurden Methoden entwickelt, bei denen keine Mesosome auftraten, die man aber aus unabhängigen Gründen für zuverlässiger hielt. (Vgl. Kap. 5, Abschn. 3d.)

Aus semantischer Sicht ist hierbei interessant, dass man nicht annahm, dass es Mesosome gar nicht gibt, sondern dass sie Artefakte sind: dass sie nicht das sind, wofür man sie hielt. So schreibt Golecki in einem Handbuch zur mikrobiologischen Elektronenmikroskopie 1988 über Mesosome:

The mesomes were defined as sac-like invaginations of the cytoplasmic membrane ... They were thought to be involved in several fundamental processes such as replication and segregation of the chromosomes, and cell division ... However, in the recent literature doubts about the classical mesosome concept have been expressed by an increasing number of reports ... [The] results of several investigations using modern cyrofixation methods ... produces evidence that the classical mesosomes are artefacts of the preparation techniques. (Golecki 1988, 73/74; meine Hervorhebung.)

Man sollte sich in dieser Passage nicht durch die Rede von Begriffen und Definitionen darin irreführen lassen, was semantisch durch die Entdeckung geschah, dass es sich um Artefakte handelt. Zentral ist die Einsicht, dass die ,klassischen Mesosome', die man lange erforscht hatte, die man für natürliche Einstülpungen der Zellmembran gehalten und als für verschiedene Prozesse verantwortlich betrachtet hatte (was zum ,Begriff', d.h. der Theorie der klassischen Mesosome gehörte), sich als Artefakte der Präparationsmethode entpuppten. Das bedeutet aber, dass der Ausdruck „Mesosom“ schon immer nur die Strukturen in präparierten Bakterien bezeichnete, die man nach wie vor auf elektronenmikroskopischen Aufnahmen identifizieren kann. Zwar hat sich die Auffassung der grundlegenden Beschaffenheit und Funktion der Mesosome geändert. Die Klasse der tatsächlichen und möglichen Strukturen, die der Begriff bezeichnet - seine Intension -, bleibt aber offenbar von dieser grundlegenden Änderung der theoretischen Auffassung der Entitäten weitgehend unberührt. Diese Stabilität der Bedeutung ist hier plausiblerweise darauf zurückführbar, dass Mesosome ein so charakteristisches Aussehen haben und dass sie daher auf elektronenmikroskopischen Aufnahmen oft ohne Hinzunahme theoretischer Annahmen zuverlässig identifiziert werden können. ${ }^{49}$

49 Siehe Hacking (1983), 179 für die vergleichbaren Behauptungen, dass Positronen theorieunabhängig auf Fotoplatten erkannt werden können und die Bedeutung von „Positron“ keine Theorie voraussetzt. 
(Es ist klar, dass sich die Zuverlässigkeit der Identifikation hier nur auf Mesosome als Strukturen in präparierten Bakterien beziehen kann. Der Präparationsprozess hat sich ja gerade als unzuverlässig in dem Sinn herausgestellt, als er die natürliche Struktur der Bakterien massiv verändert und zu Artefakten führt. ${ }^{50}$ Allerdings bezieht sich „Mesosom“ schon immer auf die Strukturen in präparierten Bakterien, und daher waren die Beobachtungen und Beobachtungsurteile über das Vorliegen von Mesosomen tatsächlich immer zuverlässig.)

Beobachtungen mit bildgebenden Verfahren sind demnach ihren Inhalten nach oft weitgehend, wenn auch nicht vollständig theorieunabhängig. Die piktorialen Inhalte von Bildern erlauben oft auf theorieunabhängige Weise verlässliche perzeptuelle Klassifikationen, die die Grundlage für den Erwerb von Begriffen für die abgebildeten Strukturen und Objekte sein können. Somit können diese Inhalte außer durch explizite, wissensbasierte Interpretationen auch durch theorieunabhängige Wahrnehmungen wissenschaftlich fruchtbar gemacht werden.

\section{b) Die Einschätzung der Verlässlichkeit}

Auch aus der Verlässlichkeitseinschätzung der Beobachtungsergebnisse ergibt sich bei bildgebenden Verfahren nur eine eingeschränkte Theorieabhängigkeit. Hierfür sprechen bei piktorialen Inhalten und den darauf beruhenden perzeptuellen Klassifikationen weitgehend dieselben Gründe, die schon bei den Instrumentewahrnehmungen vorgebracht wurden.

Zwar ist bei solchen Verfahren wie der Magnetresonanz-Tomographie offensichtlich, dass man schon gut ausgearbeitete Theorien etwa über magnetische Momente besitzen muss, um überhaupt die Instrumente konstruieren und bauen zu können. Die wissenschaftlichen Teildisziplinen, die sich mit der Entwicklung und Verbesserung solcher bildgebender Verfahren beschäftigen, sind dementsprechend voll ausgewachsene Spezialdisziplinen, die für die Entwicklung der bildgebenden Verfahren sowohl auf Grundlagentheorien zurückgreifen als auch eigene Theoriebildung betreiben. Insofern sind Beobachtungen mit diesen Verfahren sicherlich schwach theorieabhängig. Aber sobald die Verfahren entwickelt sind, können im Prinzip die Ergebnisse vor völlig verschiedenen theoretischen Hintergründen Verwendung finden. Zentral ist hierfür nur, ob die Ergebnisse vor einem solchen Hintergrund als verlässlich eingestuft werden. (Vgl. Kap. 5, Abschn. 2a.)

50 Es ist diese Unzuverlässigkeit, mit deren Aufdeckung sich die Studien von Rasmussen (1993), Culp (1994) und Hudson (1999) beschäftigen. 
Aber gerade was die piktorialen Inhalte der Bilder und darauf aufbauende Wahrnehmungen angeht, können empirische Begründungen besonders aussagekräftig sein. Die Bilder zeigen oft eine Vielzahl von Details, deren Überprüfung starke empirische Begründungen der Verlässlichkeit etwa durch induktive Erweiterungen der Beobachtungsbereiche, Koinzidenzen und kausale Analysen ergeben. (Siehe Kap. 5, Abschn. 3d[iii]-[v].) So kann die Verlässlichkeit von Computertomogrammen hinsichtlich der Abbildung anatomischer Strukturen empirisch geprüft werden, indem man die Abbildungen mit direkten Wahrnehmungen der Strukturen oder mit Abbildungen anderer Verfahren wie der Magnetresonanz-Tomographie vergleicht.

Auch bei der Einführung der funktionalen Magnetresonanz-Tomographie in die Neuroforschung spielten empirische Gründe für die Verlässlichkeit eine entscheidende Rolle. So argumentiert Kenneth Kwong in einem der ersten Aufsätze, in denen diese Möglichkeit der Beobachtung von Gehirnaktivität nachgewiesen wurde, vor allem auf zwei Weisen für die Zuverlässigkeit des Verfahrens. ${ }^{51}$ Zum ersten wird argumentiert, dass die Gehirnaktivität, die man mit Hilfe des Verfahrens bei einer visuellen Stimulation beobachtet, gut mit den Ergebnissen anderer Verfahren übereinstimmt. So erscheint der primäre visuelle Kortex, wie er sich anatomisch identifizieren lässt, als aktiv; und die maximale Aktivität ergibt sich bei einer visuellen Stimulation durch Lichtblitze mit $8 \mathrm{~Hz}$, was genau mit dem Maximum übereinstimmt, das auch die Positronen-Emissions-Tomographie - ein unabhängiges Verfahren der Beobachtung von Gehirnaktivität - ergibt. Zweitens korrelieren die Phasen, in denen der primäre visuelle Kortex als aktiv erscheint, genau mit den Phasen der Stimulation, während die beobachtete Aktivität endet, sobald der Reiz ausgesetzt wird. So ergibt sich insgesamt eine weitgehend empirische Begründung der Annahme, dass die funktionale Magnetresonanz-Tomographie verlässliche Beobachtungen der Gehirnaktivität ermöglicht.

Soweit allerdings präzise begriffliche Inhalte der Bilder betroffen sind, die auf der farblichen Kodierung gemessener Größen beruhen, ist die Verlässlichkeit oft wie bei gewöhnlichen Messgeräten einzuschätzen. Bei solchen Messgeräten übernimmt die theoretische Begründung der Verlässlichkeit typischerweise eine größere Rolle. Da die Inhalte explizit und genau spezifiziert werden, ist es in der Regel unerlässlich, exakte Annahmen über die Signale und deren Genese und Verarbeitung anzuführen. Wenn im Falle der funktionalen Magnetresonanz-Tomographie etwa der veränderte Sauerstoffgehalt des Blutes farblich dargestellt werden soll, sind genaue Annahmen über die Magnetisierung des Hämoglobins, über die Ausrichtung im homogenen

51 Siehe Kwong et al. (1992); vgl. Ogawa et al. (1992). 
Magnetfeld, die Anregung durch den gezielten Puls und das Magnetsignal, das bei der wieder parallelen Ausrichtung zustande kommt, notwendig. Hier spielen auch die Annahmen hinein, die für die Konstruktion der Instrumente verwendet wurden, und diese Annahmen sind oft theoretisch sehr voraussetzungsreich.

Beispielsweise haben theoretische Berechnungen die Glaubwürdigkeit von Aufnahmen der atomaren Struktur von Metalloberflächen mit dem Rastertunnelmikroskop in Frage gestellt. Bei solchen Mikroskopen werden die Oberflächen in der Regel mit einer feinen Wolfram-Spitze daraufhin abgetastet, in welchem Abstand ein konstanter Tunnelstrom fließt. Daraus erstellt man das Profil der Oberfläche. Die theoretische Simulation dieses Abtastvorgangs auf der Grundlage der Dichtefunktionaltheorie ergab, dass Spitze und Oberfläche sich aufgrund von atomarer Anziehung stark verformen und leicht chemische Bindungen eingehen. Demnach ist eine Abtastung der Oberfläche nur in einem eng begrenzten Abstand sinnvoll möglich, und auch in diesem Bereich führen die Anziehungskräfte dazu, dass Atome der Oberfläche gegenüber Atomzwischenräumen überhöht abgebildet werden. ${ }^{52}$ Dies illustriert, wie theoretische Gründe zunächst die Verlässlichkeit einzelner Werte für gemessene Abstände in Zweifel ziehen können. Zugleich wird aber auch unklar, was die Bilder, die auf der Grundlage dieser Messwerte erzeugt werden, insgesamt zeigen. Nur die piktorialen Inhalte - die abgebildeten Objekte und Muster und eventuelle Fähigkeiten, diese perzeptuell zu erkennen,-- bleiben von dieser Erschütterung theoriegeleiteter Interpretationen verschont.

Da solche theoretischen Annahmen bei der Verlässlichkeitseinschätzung bildgebender Verfahren oft eine größere Rolle spielen als bei Instrumentewahrnehmungen, bei denen keine explizite Kodierung von Messwerten und oft weniger theoriegeleitete Interpretation stattfinden, ist diese Einschätzung bei bildgebenden Verfahren insofern oft stärker theorieabhängig. Dies mindert aber nicht die Möglichkeiten, die Verlässlichkeit der Abbildung und der damit gemachten Beobachtungen insbesondere hinsichtlich der piktorialen Inhalte auch empirisch und damit theorieunabhängig zu begründen.

\section{c) Theoretische Annabmen in der Bildbearbeitung}

Eine weitere Theorieabhängigkeit von Beobachtungen mit bildgebenden Verfahren kann sich durch die Signalverarbeitung bei der Bildgenerierung ergeben. Hierbei fließen oft implizit oder explizit Annahmen ein, die das

52 Siehe Hofer et al. (2001). 
Beobachtungsresultat mitbestimmen. Es sind hier eine ganze Reihe unterschiedlicher Einflüsse möglich.

Beim Einsatz tomographischer Verfahren insbesondere in der medizinischen Diagnostik finden sich häufig sehr ausgeprägte Einflüsse auch von theoretischen Annahmen. Das Ziel ist hier oft, ein Bild zu generieren, in dem verschiedene anatomische Strukturen ,segmentiert ${ }^{\star}$, d.h. voneinander abgehoben dargestellt werden. Im hierbei angestrebten Idealfall werden einzelne Gewebearten, Knochen, Blutgefäße, Nervenstränge oder Tumore schon durch die farbliche Darstellung im Bild unterschieden. ${ }^{53}$ Für die Segmentierung werden eine große Vielfalt von Verfahren eingesetzt, die sich für die vorliegenden Zwecke in drei Gruppen einteilen lassen.

Zunächst gibt es eine ganze Palette von Algorithmen, mit denen man den Datensatz - etwa Zellen von Grauwerten für gemessene Röntgenabsorption - als Ganzen bearbeiten kann. Hierzu gehören Filter, die Ausreißer herausnehmen, indem sie einzelnen Volumeneinheiten des Datensatzes den Mittelwert der umgebenden Einheiten zuweisen. Zudem gibt es Kantenoperatoren, die Kanten erkennen und verstärken sollen. Schließlich gibt es eine Vielzahl von Verfahren, um Flächen gleichen Gewebes von solchen anderen Gewebes zu trennen. Hierzu zählt das sog. Keimzellenwachstumsverfahren, bei dem von einem Startpunkt aus solange benachbarte Zellen zur selben Fläche gezählt werden, bis man auf Zellen mit stark differierendem Grauwert stößt.

Diese Verfahren können im Prinzip unabhängig davon eingesetzt werden, welche Objekte untersucht werden. Sie sind als abstrakte mathematische Algorithmen programmierbar, brauchen insofern auf keine Merkmale typischer Gewebestrukturen zurückzugreifen und entsprechen oft Algorithmen, wie man sie in der menschlichen perzeptuellen Verarbeitung findet. ${ }^{54}$ Allerdings führen diese Verfahren selten allein zu zufriedenstellenden Segmentierungen. So scheitert beispielsweise die Abgrenzung von verschiedenen Geweben häufig daran, dass die Grenze nicht durchgängig durch eine Grauwertdifferenz gekennzeichnet ist. Dies führt dazu, dass mit dem Keimzellenwachstumsverfahren abgegrenzte Gebiete oft ,auslaufen', d.h. benachbarte Bereiche eingeschlossen werden. ${ }^{55}$

Abhilfe können hier die beiden weiteren Methoden schaffen. Zum einen kann man anatomisches Wissen in die Segmentierung einspeißen. Man kann etwa Wissen aus einer Datenbank hinzunehmen, in der normale anatomische Strukturen des untersuchten Bereichs gespeichert sind. Zudem greift man auf

\footnotetext{
53 Für das Folgende siehe Sakas (1999), Jendrysiak (1999), Wegner et al. (1999).

54 Siehe Jendrysiak (1999), 26.

55 Siehe Jendrysiak (1999), 27.
} 
allgemeines Wissen über bestimmte Gewebearten zurück, etwa dass Blutgefäße sich baumartig verästeln und hierbei dünner werden, oder dass dicke Nervenstränge ihre Richtung nur wenig ändern. Zum anderen kann der Benutzer des bildgebenden Verfahrens - in der Regel ein Mediziner - sein Expertenwissen einbringen, indem dieser eine grobe oder feine Segmentierung verschiedener Gewebebereiche auf der Grundlage eigenen anatomischen Wissens oder der eigenen Wahrnehmung eines vorläufigen Bildes vornimmt. ${ }^{56}$

In der medizinischen Praxis spielen die verschiedenen Verfahren oft zusammen. In einem Verfahren beispielsweise zur Segmentierung von Hirntumoren auf tomographischen Bildern beginnt der Benutzer damit, den Tumor auf dem vorläufigen Bild grob zu umkreisen. Das Programm errechnet dann die spezifischen Textureigenschaften (mittlerer Grauwert, Standardabweichung, Homogenität etc.), die für die Abbildung dieses bestimmten Tumors charakteristisch sind. Mit der so gewonnenen Information werden dann automatisch die genauen Umrisse segmentiert. Auf dem fertigen Bild wird der Tumor dann farbig vom umgebenden Gewebe hervorgehoben. ${ }^{57}$

Solche Verarbeitungsweisen können über die oben besprochene Theoriebeladenheit von Inhalt und Verlässlichkeit hinaus sicherlich zu zusätzlicher Theorieabhängigkeit führen. Erstens sind die Inhalte segmentierter Bilder weitreichender begrifflich gefasst. Es wird jetzt nicht nur die Röntgenabsorption, sondern auch die Zugehörigkeit zu anatomischen Strukturen angezeigt. Der Begriffsbesitz für einige dieser Strukturen könnte selbst eine Theorie voraussetzen.

Zweitens ist das Beobachtungsergebnis in vielen Hinsichten stark von den implizit oder explizit einfließenden Annahmen geprägt. Nach welchen Gewebearten man überhaupt sucht und unterscheidet, richtet sich nach der gängigen Klassifikation und kann daher vor verschiedenen theoretischen Hintergründen verschieden sein. Auch die Eingriffe der Mediziner sind von deren Vorbildung abhängig, und auch hier ist Theorieabhängigkeit möglich.

Allerdings muss eine solche Verarbeitung der Signale nicht in allen Fällen zu Theorieabhängigkeit führen. Viele der generellen Algorithmen der Bildbearbeitung sind unabhängig vom spezifischen Gegenstand. Die Bildung von Mittelwerten oder die Verstärkung von Konturen sind sehr häufig hilfreich, um die Abbildungsqualität von Daten zu erhöhen, die einem gewissen Rauschen unterliegen. Daher ist es unwahrscheinlich, dass diese Verfahren sich vor dem Hintergrund verschiedener theoretischer Ansätze der Physiologie

\footnotetext{
56 Siehe Jendrysiak (1999), 26.

57 Siehe Jendrysiak (1999), 27/28.
} 
oder theoretischer Erklärungen der bildgebenden Verfahren sehr unterscheiden würden.

Zudem benutzen Verfahren, die - wie das oben beschriebene Verfahren der Tumorsegmentierung - auf der Grundlage von Textureigenschaften arbeiten, keine theoretischen Annahmen. Offensichtlich macht man sich hier den Umstand zunutze, dass Tumore oft bezüglich der Abbildung mit einem bildgebenden Verfahren beobachterfreundlich sind: Sie lassen sich aufgrund von Textureigenschaften auf vorläufigen Bildern recht zuverlässig vom umgebenden Gewebe unterscheiden. Dieses Verfahren muss nicht auf theoretisches Wissen über Tumore zurückgreifen, und es braucht den Begriff des Tumors nicht vorauszusetzen.

Man kann daher ganz grob zwischen zwei Typen bildgebender Verfahren unterscheiden. Diese Unterscheidung lässt sich analog zum Gegensatz von modularer und Helmholtz'scher Theorie der Wahrnehmung treffen. Demnach sind modulare bildgebende Verfahren dadurch gekennzeichnet, dass in die Verarbeitung der Signale im Wesentlichen nur generelle Algorithmen der Bildbearbeitung (analog zu natural constraints in der Wahrnehmung) Eingang finden und dass die Segmentierung bzw. die Klassifikation allein auf Kombinationen von solchermaßen erzeugten Bildeigenschaften reagiert. Demgegenüber greifen Helmholtz'sche bildgebende Verfahren in der Signalverarbeitung und der Identifikation von Strukturen auch auf theoretisches Wissen über die angenommenen untersuchten Strukturen zurück. Es ist zu beobachten, dass eine stärker Helmholtz'sche Bildbearbeitung zwar oft in der medizinischen Diagnostik stattfindet. Forschungsorientiert eingesetzte bildgebende Verfahren etwa in der Neuroforschung laufen aber häufiger modular ab. In diesen Fällen ist weiterhin davon auszugehen, dass die Beobachtungen recht weitreichend theorieunabhängig sind.

Damit ergibt sich für perzeptuelle Beobachtungen eine Theorieunabhängigkeit, die für verschiedene Arten der Beobachtungen typischerweise unterschiedlich weit reicht. Direkte perzeptuelle Beobachtungen für beobachterfreundliche Arten sind fast völlig theorieunabhängig möglich. Weder die perzeptuelle Verarbeitung und Klassifikation noch die Inhalte der Beobachtungen setzen hier einen theoretischen Hintergrund voraus oder laufen vor verschiedenen Hintergründen unterschiedlich ab. Die Verlässlichkeitseinschätzung kann nur in einer Minderheit der Fälle von einer Theorie abhängen, sofern diese Theorie auch empirisch optimal bestätigt sein soll. Bei Instrumentewahrnehmungen ergeben sich zwar weiter reichende theoretische Gründe für oder gegen die Verlässlichkeit. Aber auch hier sind - wie auch noch bei bildgebenden Verfahren - vielfältige rein empirische Gründe möglich, die von Theorien nur revidierbar sind, indem sie ihre eigene empiri- 
sche Stützung schwächen. Die explizit kodierten Größen bildgebender Verfahren schließlich sind zwar hinsichtlich der Inhalte und der Verlässlichkeit recht stark theorieabhängig. Zudem laufen die Signalverarbeitung und die Klassifikation in einigen dieser Verfahren helmholtzianisch ab. Aber auch noch hier sind recht theorieunabhängige Beobachtungen möglich, wenn man nämlich bei modular arbeitenden Verfahren auf die piktorialen Inhalte zugreift, die theorieunabhängige verlässliche Klassifikationen und sogar den empirischen Erwerb von Begriffen über Strukturen und Objekte ermöglichen, die der direkten Wahrnehmung nicht zugänglich sind.

Es zeigt sich damit, dass perzeptuelle Beobachtungen oft auf vielschichtige Weise sowohl theorieunabhängig als auch teilweise theorieabhängig sind. So kann eine Theorieabhängigkeit in einer Hinsicht, etwa bezüglich Teilen des Inhalts, mit Theorieunabhängigkeit sowohl in dieser Hinsicht - anderen Teilinhalten - als auch in anderen Hinsichten - etwa bezüglich der Verlässlichkeit - einhergehen. Zudem gibt es innerhalb des Spektrums perzeptueller Beobachtungen vielfältige Abstufungen der Theorieunabhängigkeit, mit typischen direkten Wahrnehmungen und helmholtzianischen bildgebenden Verfahren als Extrempunkten.

\section{Zusammenfassung}

Verschiedene Formen perzeptueller Beobachtungen haben sich als in unterschiedlichem Umfang, oft jedoch als weitgehend theorieunabhängig erwiesen. Hierfür haben sich folgende Gründe ergeben.

Vielfältige empirische Evidenz spricht dafür, dass die frühe Verarbeitung der visuellen Reize kognitiv abgeschlossen ist. Damit ist eine modulare Theorie des Sehens insgesamt empirisch besser gestützt als eine Helmholtz'sche Theorie (Abschn. 2c). Der modularen Theorie zufolge wird durch feststehende Schritte auf der Basis von Vokabular und Information, die bloß Modul-intern sind, eine Repräsentation von Oberflächen und ihrer räumlichen Relationen zum Beobachter erzeugt. Wissenschaftliche Theorien üben dann keinen Einfluss auf diese visuelle Repräsentation aus (Abschn. 2a\&b).

Auf dieser Grundlage sind für den großen Bereich der beobachterfreundlichen Eigenschaften, Arten und Individuen theorieunabhängige perzeptuelle Klassifikationen denkbar, die lokal zuverlässig sind (Abschn. 3a u. b). Viele Befunde sprechen dafür, dass wir solche Fähigkeiten zur perzeptuellen Klassifikation tatsächlich erwerben können und zum Einsatz bringen (Abschn. 3c u. d). Diese Klassifikationsfähigkeiten reichen aus, um die Bedeutung von Beobachtungsausdrücken festzulegen bzw. den Erwerb von Begriffen für die klassifizierten Eigenschaften oder Arten zu ermöglichen (Abschn. 4). Zuverlässige direkte Wahrnehmungen von beobachterfreundli- 
chen Eigenschaften, Arten und Individuen sind damit sowohl hinsichtlich der eingeschlossenen perzeptuellen Klassifikationen als auch im Wahrnehmungsurteil unabhängig von wissenschaftlichen Theorien möglich.

Diese Theorieunabhängigkeit ist - mit einigen Einschränkungen - auch bei Wahrnehmungen mit Hilfe von Instrumenten sowie bildgebenden Verfahren möglich. Auch hier sind für Arten oder Eigenschaften, die relativ zu einer Beobachtungsmethode beobachterfreundlich sind, zuverlässige Klassifikationsfähigkeiten erwerbbar, die den Besitz von Begriffen empirisch fundieren können. Für die Verlässlichkeit der Beobachtungen sind oft empirische Gründe anführbar. Einschränkungen dieser Theorieunabhängigkeit betreffen insbesondere die verstärkte Rolle theoretischer Gründe in der Verlässlichkeitseinschätzung, die theoretische Festlegung der Inhalte einzelner Bildpunkte bei bildgebenden Verfahren bzw. deren theoriegeleitete Interpretation sowie mögliche theoretische Annahmen in der zu Bildern führenden Datenverarbeitung (Abschn. 5 u. 6). 
